\title{
Ältere Arbeitslose am Scheideweg zwischen Erwerbsleben und Ruhestand: Gründe für ihren Rückzug vom Arbeitsmarkt
}

\author{
Christina Wübbeke
}

Angenommen: 27. Juli 2012 / Online publiziert: 18. September 2012

(C) Institut für Arbeitsmarkt- und Berufsforschung 2012

Zusammenfassung In Deutschland waren ab 58-jährige Erwerbslose bis zum Jahr 2007 von einem Grundprinzip aktivierender Arbeitsmarktpolitik ausgenommen: Sie hatten das Recht, Arbeitslosenunterstützung bis zur Rente zu beziehen, ohne eine neue Arbeit suchen zu müssen (Paragraph 428 SGB III). Vor dem Hintergrund der politischen Bemühungen um eine stärkere Teilhabe Älterer am Arbeitsleben beschäftigt sich der Beitrag mit der Frage, aus welchen Gründen sich die überwiegende Mehrheit der älteren Langzeitarbeitslosen mit Hilfe des ,erleichterten Leistungsbezugs“ (ELB) vom Arbeitsmarkt zurückgezogen hat. Die Untersuchung des Arbeitsmarktverhaltens dieser Gruppe anhand des ELB verringert dabei ein methodisches Problem: Da arbeitslose Bezieher von Transferleistungen dem Arbeitsmarkt zur Verfügung stehen müssen, dürften Fragen nach deren Erwerbsneigung, Konzessionsbereitschaft und Suchaktivitäten vielfach zu sozial erwünschten Antworten führen. Solche Verzerrungen sind hingegen bei denjenigen Leistungsbeziehern, denen der Gesetzgeber explizit das Recht eingeräumt hat, sich vom Arbeitsmarkt abzuwenden, in geringerem Maße zu erwarten. Die empirische Analyse zeigt auf Basis einer Befragung von Beziehern des Arbeitslosengelds II, dass mangelnde Arbeitswilligkeit nur selten der Grund für den Wechsel in den Vorruhestand war. Besonders Personen mit einer unzureichenden Absicherung für das Alter wollten meist noch arbeiten. Stattdessen begründete die Mehrheit der Betroffenen ihre Entscheidung mit der Einschätzung, den beruflichen Anforderungen nicht mehr gewachsen zu sein, keine Beschäftigungschancen mehr zu

C. Wübbeke $(\varangle)$

Institut für Arbeitsmarkt- und Berufsforschung (IAB) der

Bundesagentur für Arbeit (BA), Regensburger Straße 104, 90478

Nürnberg, Deutschland

e-mail: christina.wuebbeke@iab.de haben und auch von der Arbeitsvermittlung keine ausreichende Hilfe zu erhalten. Der Rückzug in den Vorruhestand lässt sich somit nicht in erster Linie auf nutzenmaximierende Entscheidungen zugunsten von Freizeit und gegen Erwerbsarbeit zurückführen, sondern ist insbesondere bei Frauen in Ostdeutschland vorrangig Ausdruck fehlender Erwerbsperspektiven.

Schlüsselwörter Ältere Arbeitnehmer · Arbeitslosigkeit . Erwerbsbeteiligung · Arbeitsangebot · Ruhestand .

Aktivierende Arbeitsmarktpolitik · Grundsicherung für Arbeitsuchende

JEL Klassifikationen J14 · J22 · J26 · J64 · J65 - J68

Older unemployed persons at the crossroads between working life and retirement: their motives for leaving the labour force Abstract In Germany, older unemployed people aged 58 or
more years have been exempt from a fundamental princi-
ple of activating labour market policy until 2007: They have
been entitled to unemployment benefit payments until tak-
ing up retirement pension without having to seek new em-
ployment (Section 428 of Social Code III). In light of the
political goal of an increased labour market participation of
older workers this paper deals with the reasons why the ma-
jority of older long-term unemployed people left the labour
force by making use of the Facilitated Receipt of Benefits
(FRB). Surveying the labour-market behaviour of this group
against the background of the FRB diminishes a common
methodological problem: unemployed benefit payment re-
cipients usually must be available for employment; there-
fore questions about their inclination to work, their readiness
to compromise and their job-search behaviour will probably 
lead to socially desirable answers. These distortions can be expected to be less relevant with FRB payment recipients, who are legally entitled to cease seeking employment. The empirical analysis based on a survey of recipients of the Unemployment Benefit II shows that low or no propensity to work was rarely the motive for leaving the labour market; in particular, those anticipating a low retirement income actually wanted to be re-employed. The vast majority gave three reasons for the decision to retire: an inability to cope with requirements of available jobs, a lack of job opportunities, and an absence of proper support from the public employment agency. Thus the withdrawal of older long-term unemployed people into pre-retirement cannot be attributed to a utility-maximising decision in favour of leisure and against gainful employment, but is the primary result of lacking reemployment prospects particularly for women in East Germany.

Keywords Older workers - Unemployment · Labour force participation - Labour supply · Retirement - Activating labour market policy $\cdot$ Basic income support for jobseekers

\section{Grenzen der Aktivierung älterer Arbeitsloser}

Die gesellschaftlichen Bilder vom Alter und die an Ältere gerichteten Verhaltenserwartungen haben sich in Deutschland innerhalb der letzten zwei Dekaden radikal gewandelt: Galt es bis in die 1990er Jahre allgemein als legitim, dass sich Ältere frühzeitig in den, wohlverdienten ' und entpflichteten Ruhestand zurückziehen - nach dem Motto „der Jugend gehört die Bildung und Ausbildung, dem Erwachsenenalter die Arbeit und dem Alter die Freizeit" (Lehr 2008, S. 5) -, wird von ihnen heute erwartet, möglichst lange aktiv in Wirtschaft und Gesellschaft tätig zu sein (Knopf 2002). Wurde einst der frühe Ausstieg aus der Erwerbsarbeit und ihren Belastungen von der Mehrheit der Gesellschaft als sozialer Fortschritt und Beitrag zur Humanisierung des Arbeitslebens angesehen, bestimmt heute zunehmend das Leitbild des ,aktiven Alterns“ - die möglichst lange Teilhabe am Arbeitsleben, das sinnvolle Tätigsein, Gebrauchtwerden und Dazugehören bis ins hohe Alter - die Vorstellungen vom guten, gesunden und menschengerechten Altern. Waren Ältere vormals aufgefordert, ihre Arbeitsplätze möglichst frühzeitig für Jüngere zu räumen und den Arbeitsmarkt durch ihren Rückzug ,sozialverträglich ' zu entlasten, werden sie nun nach weit verbreiteter Einschätzung zumindest in längerfristiger Perspektive dringend gebraucht, weil sie einen immer größeren Teil der Erwerbsbevölkerung und der Belegschaften stellen und jüngere Nachwuchskräfte knapper werden (kritisch dazu u.a. Roberts 2006, S. 6971). Während lange Zeit das Defizitmodell des Alters die gesellschaftliche Sichtweise auf die Älteren prägte und insbesondere die betriebliche Personalpolitik bestimmte, formt nun in wachsendem Maße das (ebenfalls einseitige und vereinfachende) Bild der ,jungen Alten“ die gesellschaftliche Wahrnehmung sowie den politischen (weniger oft noch den betrieblichen) Umgang mit dieser Gruppe (van Dyk und Lessenich 2009): „Es sind die jungen, aktiven, geistig mobilen, kontaktreichen, kommunikativen, gesunden, körperlich fitten und sportlichen, mitunter auch politisch aufmüpfigen Alten (...)“ (Dieck und Naegele 1993, S. 43), deren Erfahrungswissen und Fähigkeiten für Wirtschaft und Gesellschaft unverzichtbar seien. Verfolgte die Politik bei der Steuerung des Altersübergangs vor allem in den 1980er Jahren primär das arbeitsmarktpolitische Ziel der Verringerung des Arbeitsangebots, stehen spätestens seit Mitte der 1990er Jahre die bedeutenden Finanzierungsprobleme der sozialen Sicherungssysteme im Vordergrund, welche zunächst aus der enormen Zunahme von Frühverrentungen und der hohen Arbeitslosigkeit nach der Wiedervereinigung resultierten, sich in Zukunft aber mehr und mehr (auch) aus der gesellschaftlichen Alterung ergeben werden. So erscheint es weiten Teilen der Politik und der Wissenschaft als unabdingbar, dass die Älteren einen Teil ihrer hinzugewonnenen gesunden Lebensjahre für eine längere Erwerbsbeteiligung nutzen und damit ihren Beitrag zur Entlastung der sozialen Sicherungssysteme leisten. Entsprechend wurden die Weichen in der Sozial- und Arbeitsmarktpolitik inzwischen längst in Richtung einer längeren Lebensarbeitszeit gestellt: Rascher und umfassender als in vielen anderen europäischen Ländern wurden in Deutschland seit Anfang der 1990er Jahre die Möglichkeiten eines sozial abgesicherten vorzeitigen Übergangs in den Ruhestand schrittweise beschnitten (Kraatz et al. 2006) und so der frühzeitige Ausstieg für Betriebe und vor allem die Älteren selbst erheblich verteuert.

Ein beträchtlicher Teil der älteren Arbeitnehmer hat auf die veränderten Rahmenbedingungen reagiert und bleibt nun länger im Beruf, um hohe Rentenabschläge zu vermeiden; dazu zählen insbesondere Ältere in Berufen mit vergleichsweise gemäßigten Arbeitsbelastungen. Für andere führt die Politik der Lebensarbeitszeitverlängerung jedoch zu längeren und prekäreren Statuspassagen vom Erwerbsleben in den Rentenbezug, verbunden mit erheblichen Einbußen bei der gesetzlichen Rente (Brussig 2010a, S. 6, 8, 13 f.; Brussig 2010b; Brussig und Wojtkowski 2007, S. 12; Jabsen und Buchholz 2009, S. 18, 25; Mika und Baumann 2008; Motel-Klingebiel und Engstler 2008; Trischler und Kistler 2010a, S. 29-32; Trischler und Kistler 2010b). Betroffen davon sind vor allem ältere Arbeitslose - darunter wiederum überproportional viele Geringqualifizierte mit vormals gesundheitlich belastenden beruflichen Tätigkeiten -, denn ihre Reintegrationschancen in Arbeit haben sich auch unter dem Vorzeichen der neuen Aktivierungspolitik nicht grundlegend verbessert und ihr Risiko eines langen Verbleibs in Arbeitslosigkeit ist nach wie vor besonders hoch (Statistik 
der Bundesagentur für Arbeit 2011, S. 35 f.; zur altersspezifischen Verweildauer im Arbeitslosengeld-II-Bezug: Statistik der Bundesagentur für Arbeit 2010, S. 12, 15 f.; siehe auch Brussig 2011; Walwei 2010, S. 422 f.).

Die offensichtlichen Grenzen einer Aktivierung und beruflichen Eingliederung dieser Gruppe manifestieren sich in einem politischen Zugeständnis, das von 1986 bis Ende 2007 überdauerte: Bezieher von Arbeitslosengeld, Arbeitslosenhilfe oder Arbeitslosengeld II ab dem Alter von 58 Jahren konnten in diesem Zeitraum für den so genannten „Leistungsbezug unter erleichterten Voraussetzungen“ (ELB) nach Paragraph 428 Sozialgesetzbuch (SGB) III optieren: Sie konnten erklären, dass sie keine Arbeit mehr aufnehmen wollten, und waren dann von der Verpflichtung zur Arbeitssuche befreit. Da sie nicht mehr als arbeitslos galten, blieben ihnen arbeitsmarktpolitische Maßnahmen, die diesen Status voraussetzten, verschlossen. Zudem mussten sie in Altersrente wechseln, sobald sie einen Anspruch auf eine abschlagsfreie Rente erlangt hatten. Weitere Auflagen oder Einschränkungen gab es für die betroffenen älteren Leistungsbezieher nicht.

In den letzten Jahren vor dem Auslaufen der Regelung des Paragraphen 428 SGB III nutzte jeweils eine große Mehrheit der Anspruchsberechtigten diese Ausstiegsoption. ${ }^{1}$ Vor dem Hintergrund der politischen Bemühungen um eine stärkere Teilhabe Älterer am Arbeitsleben und der veränderten gesellschaftlichen Verhaltenserwartungen ihnen gegenüber beschäftigt sich dieser Beitrag mit den Beweggründen für den Rückzug vom Arbeitsmarkt: Präferierten die meisten Betroffenen Freizeit gegenüber der Erwerbsarbeit, weil ihnen Transferzahlungen während der Arbeitslosigkeit und der nahende Renteneintritt eine finanziell akzeptable Alternative zum Angebot ihrer Arbeitskraft zum Marktlohnsatz boten? Oder sah die überwiegende Zahl der Älteren für sich keine Chance mehr auf dem Arbeitsmarkt und zog sich aus Resignation und Perspektivlosigkeit zurück? Aus den Antworten auf diese Fragen ergeben sich jeweils spezifische Folgerungen für die Arbeitsmarktpolitik gegenüber Älteren.

Aus methodischer Sicht bietet die Untersuchung der Erwerbsorientierung älterer Arbeitsloser anhand der Institution des ELB einen besonderen Vorteil. So dürften arbeitslose Bezieher von Transferleistungen, die dem Arbeitsmarkt zur Verfügung stehen müssen, bei Fragen nach ihrer Erwerbsneigung, Konzessionsbereitschaft und Suchaktivitäten in hohem Maße zu sozial erwünschten Antworten tendieren

\footnotetext{
${ }^{1}$ Im Jahr 2005 machten durchschnittlich $83 \%$ (233.000) der anspruchsberechtigten Bezieher des Arbeitslosengelds und geschätzte 160.000 Empfänger des Arbeitslosengelds II Gebrauch von dieser Regelung (Bundesagentur für Arbeit 2006, S. 142 und 2007, S. 123). In den darauf folgenden Jahren blieb die Inanspruchnahme etwa auf diesem Niveau oder überstieg es sogar (Brussig und Wübbeke 2009, S. 257 f.).
}

(siehe Krumpal und Näher 2012). Solche Verzerrungen sind hingegen bei denjenigen Leistungsbeziehern, denen der Gesetzgeber explizit das Recht eingeräumt hat, nicht mehr arbeiten zu wollen, in geringerem Maße zu erwarten. Indizien dafür liefern die Ergebnisse einer zusätzlichen Auswertung der für diese Untersuchung verwendeten Befragungsdaten. Dazu wurden die ab 58-jährigen ELB-Berechtigten (Nutzer und Nichtnutzer des ELB) mit 56- und 57-jährigen Leistungsbeziehern ohne Anspruch auf den ELB verglichen - in Hinblick auf die Arbeitssuche in den letzten vier Wochen vor dem Interview, die Gründe für unterlassene Arbeitssuche und die Motive für die Kontaktaufnahme mit dem Jobcenter. Insgesamt zeigte sich in der Gruppe der Befragten mit legaler Vorruhestandsoption ein höherer Anteil an Personen, die signalisierten, eine Rückkehr in das Erwerbsleben nicht mehr anzustreben. Dieses Ergebnis hatte auch unter Kontrolle zusätzlicher soziodemographischer, -ökonomischer und erwerbsbiographischer Faktoren Bestand. Daher ist anzunehmen, dass die im Folgenden ausgewerteten Aussagen häufiger als bei älteren Arbeitslosen ohne ELB-Option die tatsächlichen handlungsrelevanten Motive der Betroffenen und seltener lediglich gesellschaftliche Erwartungen widerspiegeln. Dennoch ist auch in der Untersuchungsgruppe mit Verzerrungen zu rechnen: Zum einen dürfte ein Teil der Befragten trotz der legalen Ausstiegsmöglichkeit ihre Arbeitsunwilligkeit als sozial unangemessen empfunden und daher nicht berichtet haben; denn im Zuge der öffentlichen Debatte um die Folgen des demographischen Wandels hat der frühzeitige Ruhestand an gesellschaftlicher Legitimität eingebüßt (siehe u.a. van Dyk und Lessenich 2009). Zum anderen beruhen die hier vorgestellten Analysen auf einer Querschnittbefragung, in der die Beweggründe für die Wahl des ELB retrospektiv erfragt wurden. Da somit Ursache und Wirkung nicht in ihrer tatsächlichen zeitlichen Reihenfolge getrennt voneinander erhoben wurden, wie dies bei Panelstudien möglich ist, bleibt unklar, in welchem Ausmaß die berichteten Handlungsmotive nachträgliche Rechtfertigungsversuche der Befragten für ein als sozial unerwünscht angesehenes Verhalten (post-hoc-Rationalisierung) oder deren tatsächliche Entscheidungsgründe abbilden (siehe auch Auspurg 2010, S. 17).

Der Beitrag untersucht die Rückzugsgründe auf Basis von Befragungsdaten, die zwischen 2005 und 2006 durch quantitative Interviews mit Beziehern des Arbeitslosengelds II gewonnen wurden. Daher beschränkt sich die empirische Analyse auf eine Teilgruppe der Adressaten des ELB: die meist langzeitarbeitslosen Älteren in der Grundsicherung für Arbeitsuchende. Im Vergleich zu den älteren Arbeitslosen insgesamt kennzeichnet diese Gruppe ein besonders hoher ökonomischer Druck zur Aufnahme einer Arbeit, da die Betroffenen etwaige Ersparnisse bis auf Freigrenzen auflösen mussten, sie während des Hilfebezugs nur 
sehr geringe Anwartschaften in der gesetzlichen Rentenversicherung erwerben und für den aktuellen Lebensunterhalt lediglich das soziokulturelle Existenzminimum zur Verfügung haben. Zudem kann vermutet werden, dass die hier untersuchten Erwerbslosen ihren Arbeitsplatz nicht im Rahmen einer betrieblichen Vorruhestandsregelung aufgegeben haben, denn diese Vereinbarungen basieren typischerweise auf einer lückenlosen Abfolge von Beschäftigung, Bezug von Arbeitslosengeld und Rentenbezug. Daher dürfte die Ruhestandsorientierung von älteren Beziehern des Arbeitslosengelds II schwächer ausgeprägt sein als die der älteren Arbeitslosen insgesamt. Dieser höhere Erwerbsdruck trifft jedoch zusammen mit deutlich schlechteren Beschäftigungsaussichten aufgrund langer Erwerbslosigkeitsdauern und vielfach weiterer vermittlungshemmender Merkmale. Für die aktivierende Arbeitsmarktpolitik handelt es sich deshalb um eine besonders schwierige Gruppe.

Im Folgenden werden zunächst die möglichen Gründe für die starke Inanspruchnahme des ELB aus der Perspektive der mikroökonomischen Arbeitsangebotstheorien einerseits und der psychologisch-soziologischen Arbeitslosigkeitsforschung andererseits diskutiert (Abschn. 2). Nach der Vorstellung der verwendeten Daten und der Untersuchungsgruppe (Abschn. 3.1) werden die aus den Theorien abgeleiteten Hypothesen zunächst anhand der von den Älteren selbst berichteten Motive überprüft (Abschn. 3.2). Diese subjektiven Befunde werden ergänzt durch eine logistische Regressionsanalyse der sozioökonomischen Einflussgrößen auf die Wahl des ELB (Abschn. 3.3). Der letzte Abschnitt fasst die wichtigsten Ergebnisse zusammen und zieht Schlussfolgerungen (Abschn. 4).

\section{Erklärungsansätze}

\subsection{Die Wahl des ELB als mikroökonomische} Arbeitsangebotsentscheidung

Die mikroökonomischen Arbeitsangebotstheorien betrachten die Anbieter von Arbeitskraft als rational handelnde und nach der Maximierung ihres Nutzens strebende Individuen. Die meisten Modelle nehmen dabei an, dass Menschen typischerweise nur aus Einkommen (genauer: aus dem dadurch ermöglichten Konsum) und aus Freizeit Nutzen ziehen, während Erwerbsarbeit selbst keinen Nutzen stiftet oder sogar nutzenmindernd wirkt, wenn sie mit Arbeitsleid verbunden ist (Franz 2006, S. 49). Deshalb kann die Gewährung von Transferleistungen ohne eine daran geknüpfte zwingende Verpflichtung zur Arbeit bewirken, dass Arbeitslose nicht mehr bereit sind, zu demjenigen Marktlohn zu arbeiten, den sie gemäß ihrer Produktivität auf dem Arbeitsmarkt erzielen könnten. Dies ist dann der Fall, wenn sie den Gesamtnutzen aus Transferzahlung und Freizeit höher schätzen als den Nutzen aus dem Marktlohn vermindert um das Arbeitsleid. Dabei gehen die meisten am neoklassischen Standardmodell orientierten Arbeitsangebotstheorien grundsätzlich von freiwilliger Arbeitslosigkeit aus: Die Betroffenen könnten eine Beschäftigung finden (und wissen dies auch), wenn sie nur dazu bereit wären, zu dem ihrer Produktivität entsprechenden Marktlohnsatz zu arbeiten (Hielscher und Ochs 2009, S. 13 f.; Koch et al. 2005, insbes. S. 420 f.). Der ELB setzte somit Anreize für einen Rückzug Älterer vom Arbeitsmarkt, weil er ein Einkommen ohne Arbeit oder die Verpflichtung hierzu ermöglichte. Auf dieses Menschenbild und diese Handlungstheorie stützten sich implizit oder explizit viele Kritiker des ELB, wenn sie diesen wegen seiner negativen Arbeitsanreize als Hemmnis für die Arbeitsmarktintegration Älterer ansahen (Eichhorst 2006). Sie folgten damit der Hypothese vom freiwilligen Ruhestand.

Allerdings ist anzunehmen, dass ältere Arbeitslose ihre Arbeitsangebotsentscheidung nicht allein auf die Maximierung ihres aktuellen Nutzens abstellen, sondern auch ihre Konsummöglichkeiten in der nahen Ruhestandsphase ins Kalkül ziehen. Letzteres wird in dynamischen Arbeitsangebotsmodellen zum Übergang in den Ruhestand berücksichtigt. Danach wählen Ältere den Zeitpunkt ihres Ausstiegs aus dem Arbeitsleben idealerweise so, dass ihr Nutzen aus Erwerbs- und Renteneinkommen sowie aus Freizeit für die gesamte restliche Lebensspanne prospektiv maximiert wird. Tatsächlich konnte der Einfluss ökonomischer Kalküle auf die individuelle Ruhestandsentscheidung für viele Industrieländer empirisch nachgewiesen werden (vgl. z. B. BörschSupan 2000; Gruber und Wise 2002). Übertragen auf die Entscheidungssituation der älteren Arbeitslosen ist demnach zu erwarten, dass die Betroffenen ihre Wahl insbesondere davon abhängig machen, wie hoch ihre Anwartschaften in den verschiedenen Alterssicherungssystemen bereits sind und welche zusätzlichen Anwartschaften sie durch eine Beschäftigung zum Marktlohnsatz noch erwerben könnten im Vergleich zum Verbleib in der Arbeitslosigkeit bis zum Renteneintritt (siehe dazu Radl 2007, S. 54 f.; Drobnič 2002, S. 89-94; Wübbeke 2005, S. 219-223). Allerdings spielen für die Konsummöglichkeiten vor und in der Ruhestandsphase ebenso das Einkommen anderer Haushaltsmitglieder, andere Einkommensarten (z.B. Mieten) sowie Vermögen und Schulden des Haushalts eine Rolle (Drobnič 2002). Für die empirische Überprüfung der Hypothese vom freiwilligen Ruhestand müsste daher die gesamte Einkommens- und Vermögenssituation des Haushalts einschließlich der Altersvorsorge berücksichtigt werden. Dieser Anspruch lässt sich mit den verfügbaren Daten zwar nicht einlösen, doch wird ökonomischen Kalkülen bei der Wahl des ELB zumindest insofern Rechnung getragen, als die subjektive Einschätzung der Einkommenslage nach dem Renteneintritt und das Vorhandensein von Wohneigentum als erklärende Variablen in die multivariate Analyse einbezogen werden. 
Die Entscheidung für den ELB drückt dabei nicht unbedingt eine generelle Abkehr vom Erwerbsleben aus. Vielmehr kann die Inanspruchnahme dieser Regelung auch für die Gruppe der grundsätzlich erwerbsorientierten Arbeitslosen eine nutzenmaximierende Wahl sein. So schützt der ELB die Älteren vor dem Zwang, auch solche Jobangebote zu akzeptieren, die hinsichtlich der Art der Tätigkeit, der Entlohnung, der Arbeitsbedingungen oder der Entfernung zum Wohnort den eigenen Mindestanforderungen nicht entsprechen. Für die untersuchten Empfänger von Arbeitslosengeld II, für welche sehr rigide Kriterien in Bezug auf die Zumutbarkeit von Arbeitsstellen gelten, dürfte dieser Vorteil von erheblicher Bedeutung sein. Dies gilt insbesondere für Ältere mit langjähriger Berufserfahrung, deren Reservationslohn sich häufig auch nach längerer Arbeitslosigkeit noch an ihrem früheren Erwerbseinkommen orientiert und damit vielfach deutlich über dem Lohnniveau der von den Arbeitsvermittlern unterbreiteten Stellenangebote liegt (Bender et al. 2008). ${ }^{2}$ Zudem entbindet der ELB von weiteren mit dem Arbeitslosenstatus verknüpften Pflichten, wie dem Nachweis eigener Bemühungen zur Beendigung der Arbeitslosigkeit oder der Teilnahme an angebotenen Fördermaßnahmen. Diese Deutung wird im Folgenden als Hypothese vom Streben nach Autonomie bezeichnet.

Der bisher dargestellte ökonomische Erklärungsansatz geht davon aus, dass sich die Wahl des ELB als individuelle Arbeitsangebotsentscheidung adäquat charakterisieren lässt: Ältere wählen den ELB, wenn es für sie nicht attraktiv ist, ihre Arbeit zum Marktlohnsatz oder zu den von der Arbeitsvermittlung als zumutbar angesehenen Konditionen anzubieten. Dabei wird unterstellt, dass die Betroffenen die Rückkehr in Erwerbsarbeit auch nach längerer Arbeitslosigkeit noch als reale Handlungsoption ansehen und dass die meisten Betroffenen Freizeit einer Erwerbsarbeit vorziehen, sofern ihnen die damit verbundenen finanziellen EinbuBen als akzeptabel erscheinen. Erkenntnisse der psychologischen und soziologischen Forschung zu den individuellen Folgen lange andauernder Arbeitslosigkeit stellen die allgemeine Gültigkeit dieser Annahmen allerdings in Frage.

\footnotetext{
${ }^{2}$ Die Orientierung am früheren beruflichen Status und Lohn hat jedoch nicht nur materielle, sondern mindestens ebenso sehr psychologische Gründe. So belegen Studien, dass Menschen erhebliche Anstrengungen unternehmen, ihre autobiographisch geformte berufliche Identität auch nach dem Ausscheiden aus dem Erwerbsleben noch möglichst lange aufrechtzuerhalten. Deshalb empfinden es erwerbslose Leistungsempfänger häufig als eine Gefährdung ihres labilen Selbstbildes und als soziale Degradierung, wenn ihnen Stellen angeboten werden, die nicht an ihren früheren beruflichen Qualifikationen und Erfahrungen anknüpfen (Giddens 1991; Riach und Loretto 2009, S. 112 f.). Auf die Bedeutung psychologischer Zusammenhänge für den Rückzug Älterer vom Arbeitsmarkt wird im folgenden Abschnitt ausführlicher eingegangen.
}

2.2 Soziologisch-psychologische Deutung: Der ELB als unfreiwilliger Ruhestand

Eine umfangreiche Metaanalyse von psychologischen Studien aus verschiedenen Industrieländern konnte zeigen, dass sich Arbeitslose und Erwerbstätige hinsichtlich ihrer Einstellung zur Arbeit nur geringfügig voneinander unterscheiden (Paul und Moser 2006, insbes. S. 602-605): In beiden Gruppen wird der Erwerbsarbeit an sich - abstrahiert von der Art der Tätigkeit, ihrem spezifischen Prestige und ihrer Entlohnung - mehrheitlich ein hoher Wert zugeschrieben. Dabei ist die innere Bindung an Erwerbsarbeit offenbar eine relativ stabile Eigenschaft: Sie ändert sich kaum, wenn Menschen ihre Arbeit verlieren oder nach Arbeitslosigkeit wieder eine neue Stelle finden und bleibt selbst nach langer Arbeitslosigkeit nahezu konstant.

Gemessen wurde die Erwerbsorientierung in den Studien, die Eingang in die Metaanalyse fanden, mit Hilfe von ,scales of work involvement, Protestant work ethic or similar concepts" (Paul und Moser 2006, S. 595). Diese bilden das „psychosoziale Bedürfnis“ nach Arbeit ab, also die nichtfinanzielle Arbeitsmotivation (Nordenmark und Strandh 1999, S. 582, 586). Die durch die Studien belegte hohe Wertschätzung der bezahlten Arbeit in weiten Teilen der Erwerbsbevölkerung geht somit über die rein wirtschaftliche Bedeutung der Arbeit hinaus. Sie ist das Ergebnis eines lebenslangen Sozialisationsprozesses und spiegelt die vorherrschende Arbeitsethik in modernen erwerbsarbeitszentrierten Gesellschaften wieder sowie deren Kehrseite, die Tendenz zur Stigmatisierung von Arbeitslosen (Goffmans Stigma-Theorie 1963; Kirchler 1991, S. 84-115). Entsprechend heben prominente Arbeitslosigkeitstheorien die psychosozialen Funktionen hervor, die Erwerbsarbeit im Idealfall erfüllt (Warrs Vitamin-Modell 1987) oder die in modernen Gesellschaften sogar nur die Erwerbsarbeit vollständig erfüllen kann (Jahodas Theorie der latenten Deprivation, 1983). Dazu zählt u.a. die Bedeutung der Arbeit für die soziale Identität, den sozialen Status (Riach und Loretto 2009) und das gesellschaftliche Zugehörigkeitsgefühl des Einzelnen (Gundert und Hohendanner 2011), seine Anerkennung durch andere und seine Selbstachtung. ${ }^{3}$

\footnotetext{
${ }^{3}$ Daneben verweist Jahoda auf weitere ,latente“ Funktionen, u.a. die zeitliche Strukturierung des Alltags, soziale Kontakte jenseits des engen sozialen Umfeldes, der Zwang zur Aktivität und die Teilhabe an kollektiven Zielen, die dem Einzelnen das Gefühl eigener Nützlichkeit vermittelt. Zu den neun „Vitaminen“, die Menschen nach Warr für ihr psychisches Wohlergehen benötigen, gehören u.a. Abwechslung und die Gelegenheit, neue Erfahrungen zu sammeln, die Möglichkeit der Ausübung und Entwicklung eigener Fähigkeiten, motivierende und aktivierende externe Zielvorgaben und die Möglichkeit zur Kontrolle der eigenen Lebensbedingungen. Erwerbsarbeit ist nach Warr eine wichtige, aber nicht die einzige Quelle dieser Vitamine und sie stellt diese zudem nicht immer oder nicht immer in der richtigen Dosis zur Verfügung. Demgegenüber sieht Jahoda in der Erwerbsarbeit die einzige ge-
} 
Die starke innere Bindung an Erwerbsarbeit ist nach den Forschungsergebnissen von Paul und Moser (2006) zudem eine der Ursachen für die vielfach belegte schlechtere psychische Verfassung von Arbeitslosen im Vergleich zu Erwerbstätigen. So deuten die Resultate ihrer Metaanalyse darauf hin, dass die meisten Arbeitslosen an der Inkongruenz zwischen ihrem Erwerbswunsch und dem realisierten Grad der Zielerreichung psychisch erheblich leiden (motivationale Inkongruenz, Grawe 2004); dies gilt in besonderem Maße für Personen mit geringer Qualifikation. Auch nach längerer Arbeitslosigkeit findet - entgegen einer verbreiteten Auffassung - meist kein Gewöhnungs- und Adaptionsprozess statt, obwohl die damit verbundene Abschwächung der starken Erwerbsorientierung das Wohlbefinden der Betroffenen unter Umständen verbessern könnte (Paul und Moser 2006, S. 620 f.; siehe dazu auch Jackson und Taylor 1994, S. 563-565, sowie Nordenmark und Strandh 1999, S. 581, 584). Diese Ergebnisse stehen somit in scharfem Kontrast zu der Annahme der traditionellen ökonomischen Handlungsmodelle, wonach sich die meisten Menschen für Freizeit und gegen Erwerbsarbeit entscheiden würden, wenn ihr gewünschtes Konsumniveau auch ohne Arbeit gesichert wäre (zu ähnlichen Befunden siehe Koch et al. 2005, S. 428433). ${ }^{4}$

Zugleich belegen empirische Studien jedoch, dass zwar die große Mehrheit der Erwerbslosen unmittelbar nach dem Verlust ihrer Arbeitsstelle so schnell wie möglich wieder eine neue Beschäftigung finden will, dass aber fast alle Betroffenen bei länger andauernder Arbeitslosigkeit die Hoffnung auf eine Rückkehr in Erwerbsarbeit nach einer gewissen Zeit aufgeben. In einer methodisch herausragenden niederländischen Panel-Untersuchung aus den 1980er Jahren mit 242 ausschließlich männlichen Erwerbslosen im Alter zwischen 40 und 55 Jahren zeigte sich dieser Wandel vorwiegend innerhalb der ersten zwei bis drei Jahre nach der Entlassung (Henkens et al. 1996, S. 563 und 570-573). Die wachsende Resignation spiegelte sich im Arbeitssuchverhalten deutlich wider: Während die meisten älteren Arbeitslosen zu Beginn ernsthafte Versuche unternahmen, eine neue Stelle zu finden, verringerte sich die Intensität der Suche im Verlauf der Erwerbslosigkeit relativ schnell. Nach einem Jahr suchten

sellschaftliche Institution, die in modernen Industriegesellschaften die von ihr genannten psychosozialen Bedürfnisse des Menschen umfassend befriedigen kann (zu empirischen Untersuchungen, die Jahodas Modell stützen, vgl. Paul et al. 2009 sowie die dort genannten Studien; zur Kritik beider strukturalistischer Ansätze siehe Nordenmark und Strandh 1999, S. 578 f.; siehe zu den psychosozialen Funktionen der Erwerbsarbeit auch Promberger 2008).

${ }^{4}$ Vereinzelt weichen arbeitsangebotstheoretische Modelle jedoch auch von dieser restriktiven Prämisse ab. So berücksichtigt Sessions (1994) bei der Modellierung des individuellen Nutzenkalküls die Erkenntnisse der soziologischen und psychologischen Forschung über die Wirkungen von Arbeitslosigkeit auf die psychische Verfassung der Betroffenen. nur noch $39 \%$ der Untersuchungsteilnehmer aktiv nach Arbeit, nach fünf Jahren kein einziger mehr (Henkens et al. 1996, S. 568). ${ }^{5}$

Besonders entmutigend dürften vergebliche Bewerbungsbemühungen auf ältere Arbeitsuchende dann wirken, wenn die Betroffenen die Erfahrung gemacht haben oder auch bloß vermuten, dass Arbeitgeber sie allein aufgrund ihres fortgeschrittenen Alters ablehnen, ohne ihre individuelle Eignung und Leistungsfähigkeit näher zu prüfen. ${ }^{6}$ Neben diskriminierenden Rekrutierungspraktiken und der Dauer der Arbeitslosigkeit selbst erschweren den Älteren zudem überproportional häufig weitere Integrationshemmnisse die Rückkehr in den Beruf, vor allem gesundheitliche Beeinträchtigungen, geringe oder veraltete Qualifikationen sowie eingeschränkte räumliche Mobilität (Büttner et al. 2009, S. 37 f.). Diese multiplen Handicaps lassen die Erfolgsaussichten einer Stellensuche objektiv oder auch nur aus Sicht der Betroffenen als gering erscheinen und führen bei vielen zu der Einschätzung, den Anforderungen des Arbeitsmarktes nicht (mehr) zu genügen. ${ }^{7}$

Hinzu kommt, dass Älteren vielfach auch seitens der Arbeitsverwaltung signalisiert wird, zu den Nicht- oder Schwervermittelbaren zu gehören. So berichtet ein erheblicher Teil der älteren Erwerbslosen in Befragungen von einem Desinteresse der Arbeitsvermittler und die Mehrheit der Betroffenen gibt an, von der betreuenden Stelle nicht oder nicht ausreichend unterstützt und gefördert zu werden (Brauer und Korge 2009: 13; Büttner et al. 2009, S. 44;

\footnotetext{
${ }^{5}$ Diese Befunde stehen mit der Theorie der erlernten Hilflosigkeit (Seligman 1975) in Einklang. Demnach besteht die Gefahr, dass längere unfreiwillige Arbeitslosigkeit zu einem Gefühl des Kontrollverlustes über das eigene Leben führt: Die Betroffenen generalisieren wiederholt erfahrene Hilflosigkeit (z.B. nach vielen erfolglosen Bewerbungsversuchen) infolge eines Verstärkungslernens und gehen davon aus, dass sie durch ihr eigenes Handeln wichtige Ereignisse in ihrem Leben nicht mehr beeinflussen können. Der erfahrene Kontrollverlust zieht gemäß experimentell gewonnenen Erkenntnissen regelmäßig emotionale, kognitive und motivationale Probleme nach sich. Damit schwinden die Chancen auf eine erfolgreiche Bewerbung mit der Dauer der Arbeitslosigkeit nicht nur deshalb, weil die Betroffenen in ihren Suchanstrengungen nachlassen und Qualifikationen veralten oder verlorengehen, sondern auch weil die typischen Folgen erlernter Hilflosigkeit (wie Selbstzweifel, Gefühle der Furcht und Ohnmacht, verlangsamte Lernprozesse und nachlassende Problemlösungsfähigkeit) es ihnen erschweren, einen potentiellen Arbeitgeber von den eigenen Stärken zu überzeugen (siehe dazu auch Börner 2001, S. 85 f.; Mohr und Richter 2008, S. 29; Rodriguez 1997).

${ }^{6} \mathrm{Zu}$ den Erscheinungsformen und Wirkungen von Altersstereotypen, dem meist verdeckten „Ageism“ in deutschen Betrieben sowie den Schwierigkeiten seines empirischen Nachweises siehe Brauer 2009; siehe dazu außerdem Riach und Loretto 2009, S. 109 und Koller und Gruber 2001.

${ }^{7}$ Belege für die pessimistische Einschätzung der eigenen Wiedereingliederungschancen durch ältere Arbeitslose finden sich (bezogen auf Ostdeutschland) z.B. in Haupt et al. 2005, S. 75 f. Vgl. dazu auch ähnliche Befunde einer britischen Studie in Porcellato et al. 2010.
} 
Kistler und Hilpert 2001, S. 11; Krone 2009, S. 269 f.). ${ }^{8}$ Auch die weit unterdurchschnittlichen Partizipation Älterer an arbeitsmarktpolitischen Maßnahmen in Deutschland deutet darauf hin, dass bei der Betreuung älterer Erwerbsloser vielfach nicht mehr auf deren Wiedereingliederung in den Arbeitsmarkt gesetzt wird (Frerichs und Taylor 2005; Heinemann et al. 2006).

Für die meisten älteren Arbeitslosen dürfte daher die Perspektive einer Rückkehr in das Berufsleben im Verlauf ihrer Erwerbslosigkeit zunehmend in den Hintergrund treten. Sie sehen sich folglich nicht (mehr) vor eine Arbeitsangebotsentscheidung gestellt, sondern vor die Wahl zwischen dem Verbleib in Langzeitarbeitslosigkeit und dem Rückzug in den Ruhestand. In dieser Situation dürften sich Ältere gerade wegen ihrer regelmäßig starken Erwerbsorientierung meist für den ELB entschieden haben: zum einen, weil der Wechsel in die gesellschaftliche Alternativrolle des Ruheständlers es den Betroffenen erleichtern dürfte, das Ende ihrer Erwerbskarriere zu akzeptieren und sich neuen Lebensinhalten zu widmen (Hetschko et al. 2011) ${ }^{9}$ zum anderen aber auch, weil der Status des Vorruheständlers gesellschaftlich eher akzeptiert ist als der stigmatisierende Status des Langzeitarbeitslosen (Brauer und Clemens 2009, S. 29). Da die Wahl des ELB aus dieser Sicht vorrangig fehlenden Erwerbsperspektiven geschuldet ist, lässt sich diese Interpretation als Hypothese vom unfreiwilligen Ruhestand bezeichnen.

\section{Empirische Analyse}

\subsection{Daten und Untersuchungspopulation}

Grundlage der folgenden Analysen bildet die Querschnittbefragung „Lebenssituation und soziale Sicherung 2005“,

\footnotetext{
${ }^{8}$ Demgegenüber charakterisierten ältere Bezieher von Arbeitslosengeld II in einer aktuellen Studie ihr Verhältnis zu ihren Beratern überwiegend als freundlich und vertrauensvoll, räumten deren Bemühungen aber deutlich geringere Erfolgschancen ein als die befragten jüngeren Hilfeempfänger (Tisch 2010).

${ }^{9}$ Der Wechsel in die Rolle des Ruheständlers beendet die motivationale Inkongruenz zwischen Erwerbswunsch und fehlender Erwerbsmöglichkeit und kann daher nach dem transaktionalen Stress-Modell von Lazarus als eine palliative (lindernde) Bewältigungsform der psychischen Belastungen durch Arbeitslosigkeit gedeutet werden (Lazarus und Launier 1981, S. 245-258). Empirische Belege für die Verbesserung des psychischen Wohlbefindens älterer männlicher Arbeitsloser durch die persönliche Redefinition als Ruheständler finden sich in Jackson und Taylor 1994, S. 560-562. Auch die Ergebnisse eines qualitativen Forschungsprojektes zur Erwerbsorientierung von Empfängern des Arbeitslosengelds II deuten darauf hin, dass Arbeitslose, die ,aufgrund ihres Alters (meist über 45 Jahre) oder einer Erkrankung für sich nur noch geringe Chancen auf eine reguläre Beschäftigung mit ausreichendem Einkommen“ sehen, ,nach einem gesellschaftlich anerkannten Ausstieg aus der Arbeitslosigkeit und dem damit verknüpften Aktivierungssystem“" suchen (Bescherer et al. 2008, S. 22 f.).
}

die im Zeitraum von November 2005 bis März 2006 im Auftrag des Instituts für Arbeitsmarkt- und Berufsforschung (IAB) bundesweit durchgeführt wurde. Ziel der Studie war es, die Veränderungen zu untersuchen, die mit dem Übergang vom bisherigen System der Arbeitslosen- und Sozialhilfe zum neuen System einer einheitlichen Grundsicherung für erwerbsfähige Hilfebedürftige verbunden waren. Die Grundgesamtheit der IAB-Querschnittbefragung umfasst Arbeitslosengeld-II-Empfänger vom Januar 2005 sowie Personen, die im Dezember 2004 Arbeitslosenhilfe erhalten hatten, jedoch nicht die Anspruchsvoraussetzungen für das neue Arbeitslosengeld II erfüllten. In der Hauptstudie wurden in überwiegend telefonischen, teils aber auch persönlichen Interviews insgesamt 20.832 Personen im Alter zwischen 15 und unter 65 Jahren befragt. Als Folge von lückenhaften Adressdaten zum Ziehungszeitpunkt konnten die Arbeitslosengeld-II-Bezieher vom Januar 2005 nur in 266 von 439 Kreisen der Bundesrepublik vollständig in die Studie einbezogen werden. In den übrigen 173 Kreisen, zu denen alle optierenden Kommunen gehören, beschränkt sich die Erhebung auf die Gruppe der ehemaligen Arbeitslosenhilfeempfänger, so dass in diesen Kreisen vor allem frühere Bezieher von Sozialhilfe in der Studie fehlen.

Für die nachfolgenden Analysen wurden alle Studienteilnehmer ausgewählt, die für den ELB optieren konnten, also Befragte, die nach Vollendung des 58. Lebensjahres zu irgendeinem Zeitpunkt Arbeitslosengeld oder Arbeitslosenhilfe (bis 2004) bzw. Arbeitslosengeld I oder Arbeitslosengeld II (ab 2005) bezogen hatten. Um eine Verzerrung der Ergebnisse zu vermeiden, beschränken sich die Auswertungen zudem auf diejenigen $266 \mathrm{Kreise}$, in denen die Arbeitslosengeld-II-Empfänger vom Januar 2005 vollständig in der Studie erfasst sind. Wie zusätzliche Auswertungen zeigen, veränderte der Ausschluss der unvollständigen Kreise die inhaltlichen Ergebnisse nur marginal. Da aber als Folge der Selektion ostdeutsche Hilfebezieher in der Untersuchungspopulation deutlich überrepräsentiert sind, wurden die deskriptiven Analysen jeweils für die neuen und alten Bundesländer getrennt durchgeführt. Insgesamt machten $70 \%$ der ostdeutschen und rund die Hälfte der westdeutschen Befragten aus der Untersuchungsgruppe von der Möglichkeit des ELB Gebrauch.

Als Folge der Arbeitsmarktreform ist die Untersuchungsgruppe sehr heterogen und umfasst Personen, die aus unterschiedlichen Systemen der sozialen Sicherung in den Arbeitslosengeld-II-Bezug gewechselt sind. Die größte Gruppe stellen dabei die ehemaligen Bezieher von Arbeitslosenhilfe dar, deren Anteil in Westdeutschland mit $63 \%$ deutlich geringer ist als in Ostdeutschland mit $75 \%$ (siehe Tab. 1). Hingegen machen die ehemaligen Sozialhilfeempfänger nur einen Anteil von $7 \%$ in den neuen und $21 \%$ in den alten Ländern aus. Etwa gleich stark vertreten sind in 
Tab. 1 Die Untersuchungspopulation: Arbeitslosengeld-II-Bezieher von Januar 2005 mit der Wahlmöglichkeit des ELB

\begin{tabular}{|c|c|c|c|c|c|c|}
\hline \multirow[t]{2}{*}{$\begin{array}{l}\text { Leistungsbezug im } \\
\text { Dezember } 2004\end{array}$} & \multirow[t]{2}{*}{ Anzahl } & \multirow[t]{2}{*}{ Prozent } & \multicolumn{4}{|c|}{$\begin{array}{l}\text { Dauer seit Ende der letzten mehr als geringfügigen, } \\
\text { ungeförderten Erwerbstätigkeit in Jahren }\end{array}$} \\
\hline & & & $\begin{array}{l}\text { Anzahl der } \\
\text { Fälle mit } \\
\text { gültiger Dauer }\end{array}$ & $25 \%$-Perzentil & Median & $75 \%$-Perzentil \\
\hline
\end{tabular}

\section{Westdeutschland}

\begin{tabular}{|c|c|c|c|c|c|c|}
\hline Arbeitslosen- oder Unterhaltsgeld & 69 & 11 & 68 & 0,3 & 2,8 & 6,7 \\
\hline Arbeitslosenhilfe & 415 & 63 & 410 & 1,7 & 4,1 & 7,5 \\
\hline Sozialhilfe $^{\mathrm{a}}$ & 140 & 21 & 112 & 4,1 & 8,8 & 23,2 \\
\hline Nichts davon & 30 & 5 & 24 & 0,2 & 1,1 & 9,3 \\
\hline Insgesamt & 654 & 100 & 614 & 1,4 & 4,2 & 8,5 \\
\hline \multicolumn{7}{|l|}{ Ostdeutschland } \\
\hline Arbeitslosen- oder Unterhaltsgeld & 64 & 14 & 62 & 0,2 & 1,5 & 3,7 \\
\hline Arbeitslosenhilfe & 350 & 75 & 341 & 2,2 & 4,6 & 8,7 \\
\hline Sozialhilfe $\mathrm{a}^{\mathrm{a}}$ & 34 & 7 & 31 & 1,3 & 5,4 & 11,5 \\
\hline Nichts davon & 18 & 4 & 18 & 0,2 & 4,7 & 10,5 \\
\hline Insgesamt & 466 & 100 & 452 & 1,4 & 4,2 & 8,1 \\
\hline Ost und West gesamt & 1.120 & 100 & 1.066 & 1,4 & 4,2 & 8,3 \\
\hline
\end{tabular}

Quelle: IAB-Querschnittbefragung „Lebenssituation und Soziale Sicherung 2005“(QS I); gewichtet; eigene Berechnungen

${ }^{a}$ Ohne Personen, die Sozialhilfe ergänzend zu Arbeitslosengeld, Unterhaltsgeld oder Arbeitslosenhilfe bezogen haben

beiden Landesteilen mit $11 \%$ bzw. $14 \%$ diejenigen Befragten, die im Dezember 2004 Arbeitslosen- oder Unterhaltsgeld aus der Arbeitslosenversicherung erhielten. Rund $4 \%$ der Befragten schließlich bezogen vor der Reform keine der genannten Leistungen.

Die unterschiedliche Herkunft der Befragten spiegelt sich auch in der Dauer der Ausgliederung aus regulärer Erwerbstätigkeit zum Zeitpunkt der Entstehung des Anspruchs auf den ELB wider. Tabelle 1 zeigt die Quartilswerte dieser Dauer getrennt für Ost- und Westdeutsche und differenziert nach der Art der im Dezember 2004 bezogenen Leistung. Die längsten Phasen der Ausgliederung durchliefen demnach in beiden Landesteilen die ehemaligen Bezieher von Sozialhilfe: Für die Hälfte von ihnen endete ihre reguläre Beschäftigung bereits vor mindestens 8,8 Jahren (West) bzw. 5,4 Jahren (Ost). Die kürzesten Ausgliederungsdauern weisen hingegen in West- und Ostdeutschland jeweils die ehemaligen Empfänger von Arbeitslosen- oder Unterhaltsgeld auf. Für sie ergibt sich ein Medianwert von 2,8 Jahren (West) bzw. 1,5 Jahren (Ost). Dazwischen liegen mit Medianwerten von 4,1 Jahren (West) bzw. 4,6 Jahren (Ost) die Ausgliederungsdauern der vormaligen Arbeitslosenhilfeempfänger. Insgesamt kennzeichnet die untersuchte Gruppe also eine relativ starke Arbeitsmarktferne: Die Hälfte aller ab 58-jährigen Befragten waren bereits mindestens 4,2 Jahre nicht mehr sozialversicherungspflichtig beschäftigt oder mehr als geringfügig selbständig tätig gewesen, als sich ihnen die Möglichkeit bot, für den ELB zu optieren.

\subsection{Die Motive für die Wahl des ELB nach Auskunft der Nutzer}

Die Gründe für den Rückzug vom Arbeitsmarkt werden im Folgenden zunächst anhand der selbst berichteten Motive der Betroffenen untersucht. Diese Motive wurden im Rahmen der IAB-Befragung standardisiert erhoben, indem den befragten Nutzern des ELB mögliche Beweggründe genannt wurden, die sie jeweils als zutreffend oder nicht zutreffend einstufen konnten. Die einzelnen Items lassen sich zu vier Kategorien zusammenfassen: mangelnde Arbeitswilligkeit, mangelnde Erwerbsfähigkeit, der Wunsch nach Autonomie sowie fehlende Stellen- oder Förderangebote seitens der betreuenden Stelle.

Wie Tab. 2 zeigt, begründeten nur sehr wenige Befragte ihre Entscheidung für den ELB mit dem naheliegenden Motiv: der fehlenden Bereitschaft zur Rückkehr in Beschäftigung. So gaben lediglich $13 \%$ der Westdeutschen und $9 \%$ der Ostdeutschen an, dass sie nicht mehr arbeiten wollten. Dieses Ergebnis steht in Kontrast zur politischen Begründung des ELB, nämlich der Entlastung der Arbeitslosenstatistik und Arbeitsvermittlung von solchen Erwerbslosen, die aufgrund ihres Alters keine Beschäftigung mehr anstreben und daher nicht mehr vermittelt werden wollen. Zugleich spricht der Befund gegen die Hypothese vom freiwilligen Ruhestand: Für die meisten Betroffenen scheint der Rückzug in den ELB nicht das Ergebnis einer nutzenmaximierenden Entscheidung für Freizeit und gegen Erwerbsarbeit gewesen zu sein. 
Tab. 2 Selbst berichtete Motive für die Inanspruchnahme des ELB

\begin{tabular}{|c|c|c|c|c|}
\hline \multirow{3}{*}{$\begin{array}{l}\text { Beweggründe } \\
\text { A. Fehlende Arbeitswilligkeit (Item 1) }\end{array}$} & \multirow{2}{*}{\multicolumn{3}{|c|}{$\begin{array}{l}\text { Westdeutschland } \\
\text {,Ja“-Antworten in \% } \\
\text { [95\%-Konfidenzintervall] }\end{array}$}} & Ostdeutschland \\
\hline & & & & \\
\hline & 13 & [9-17] & 9 & [5-12] \\
\hline (1) „Ich wollte nicht mehr arbeiten“ & 13 & [9-17] & 9 & {$[5-12]$} \\
\hline B. Fehlende Erwerbsfähigkeit (Item 2 oder 3) & 45 & [39-52] & 31 & [26-37] \\
\hline (2) „Ich konnte aus gesundheitlichen Gründen nicht mehr arbeiten“ & 36 & {$[30-42]$} & 25 & [20-30] \\
\hline (3) „Ich fühlte mich den Anforderungen im Beruf nicht mehr gewachsen“ & 31 & [26-37] & 16 & [12-21] \\
\hline C. Keine Hilfe von der betreuenden Stelle (Item 4 oder 5) & 96 & [93-98] & 94 & [92-97] \\
\hline (4) „Die Agentur oder ARGE konnte mir ohnehin keine Stelle vermitteln“ & 94 & [90-97] & 93 & [90-96] \\
\hline (5) „Die Agentur oder ARGE wollte mir keine Maßnahmen anbieten“ & 52 & [46-59] & 55 & [49-61] \\
\hline D. Wunsch nach Autonomie (Item 6 oder 7) & 50 & [44-57] & 50 & [44-56] \\
\hline (6) „Ich wollte nicht jede beliebige Arbeit annehmen müssen“ & 40 & {$[34-46]$} & 36 & [30-42] \\
\hline (7) „Ich wollte mich nicht laufend bewerben oder an Fördermaßnahmen teilnehmen müssen“ & 33 & [27-39] & 32 & [26-37] \\
\hline Zahl der Nutzer mit gültigen Angaben & 335 & & 319 & \\
\hline
\end{tabular}

Quelle: IAB-Querschnittbefragung „Lebenssituation und Soziale Sicherung 2005“(QS I); gewichtet; eigene Berechnungen

Tab. 3 Typisierung der Nutzer des ELB anhand der von ihnen angegebenen Motive

\begin{tabular}{|c|c|c|c|c|c|}
\hline \multirow{3}{*}{$\begin{array}{l}\text { Nutzertypen } \\
\text { Typ 1: freiwilliger Ruhestand }\end{array}$} & \multirow{3}{*}{$\begin{array}{l}\text { Zustimmung zu } \\
\text { folgenden Items }\end{array}$} & \multirow{2}{*}{\multicolumn{2}{|c|}{$\begin{array}{l}\text { Westdeutschland } \\
\text { in } \% \text { der Nutzer } \\
{[95 \% \text {-Konfidenzin }}\end{array}$}} & \multicolumn{2}{|c|}{ Ostdeutschland } \\
\hline & & & & all] & \\
\hline & & 13 & [9-17] & 9 & {$[5-12]$} \\
\hline $\begin{array}{l}\text { Typ 2: unfreiwilliger Ruhestand begründet durch subjektive } \\
\text { Erwerbsunfähigkeit }\end{array}$ & Item 2 oder 3 , nicht Item 1 & 39 & [32-45] & 27 & [22-33] \\
\hline $\begin{array}{l}\text { Typ 3: unfreiwilliger Ruhestand begründet durch fehlende } \\
\text { Stellen- und Förderangebote }\end{array}$ & ausschließlich Item 4 oder 5 & 23 & [18-29] & 36 & [31-42] \\
\hline Typ 4: Streben nach Autonomie & Item 6 oder 7, nicht Items 1 bis 3 & 25 & [20-31] & 28 & [22-33] \\
\hline Insgesamt & & 100 & & 100 & \\
\hline Zahl der Nutzer mit gültigen Angaben & & 335 & & 319 & \\
\hline
\end{tabular}

Quelle: IAB-Querschnittbefragung „Lebenssituation und Soziale Sicherung 2005“(QS I); gewichtet; eigene Berechnungen

Stattdessen spielten gesundheitliche Probleme und das Gefühl, den Anforderungen im Beruf nicht mehr gewachsen zu sein, eine erhebliche Rolle (Tab. 2). Mindestens eines der beiden Motive, die subjektiv empfundene Einschränkungen der Erwerbsfähigkeit widerspiegeln, nannten $45 \%$ der westdeutschen und $31 \%$ der ostdeutschen Nutzer. Schließt man aus dieser Gruppe diejenigen Befragten aus, die nach eigenen Angaben zugleich auch nicht mehr arbeiten wollten, so verbleiben $39 \%$ der Nutzer in West- und $27 \%$ in Ostdeutschland (Tab. 3). Diese Personen wählten den ELB offenbar in erster Linie, weil sie sich zur Erwerbsarbeit nicht mehr in der Lage sahen.

Noch häufiger scheint die Wahl des ELB aber eine Reaktion auf mangelnde Unterstützung und fehlende Beschäftigungsmöglichkeiten gewesen zu sein. So gaben nahezu alle befragten Nutzer an, dass die zuständige Agentur oder
$\mathrm{ARGE}^{10}$ ihnen ohnehin keine Stelle vermitteln konnte, und jeweils mehr als die Hälfte der Nutzer in Ost- und Westdeutschland stimmten der Aussage zu, dass ihnen die betreuende Stelle keine Fördermaßnahmen anbieten wollte (Tab. 2). Immerhin $23 \%$ der West- und $36 \%$ der Ostdeutschen nannten fehlende Stellen- oder Förderangebote sogar als einzigen Grund für ihren Rückzug (Tab. 3). Zählt man zu dieser Gruppe diejenigen Älteren hinzu, die sich aus gesundheitlichen oder anderen Gründen den Anforderungen einer beruflichen Tätigkeit nicht mehr gewachsen fühlten, obwohl sie gerne noch gearbeitet hätten, lassen sich $62 \%$ der Westdeutschen und $63 \%$ der Ostdeutschen zur Gruppe

\footnotetext{
${ }^{10}$ Der Einfachheit halber wurde in der Befragung die Bezeichnung ARGE (Abkürzung für Arbeitsgemeinschaft) stellvertretend für alle Träger der Grundsicherung für Arbeitsuchende verwendet.
} 
jener Befragten rechnen, deren Rückzug in den Ruhestand wohl unfreiwillig erfolgte (Typen 2 und 3 in Tab. 3). ${ }^{11}$

Die beiden letzten Items betreffen den Wunsch nach $\mathrm{Au}$ tonomie gegenüber der Arbeitsagentur oder ARGE: Mit dem Vorteil, nicht mehr jede Arbeit annehmen zu müssen, begründeten $40 \%$ der Westdeutschen und fast ebenso viele Ostdeutsche (36\%) ihre Entscheidung (Tab. 2). Zudem wollte jeweils ungefähr ein Drittel der Befragten in beiden Landesteilen nicht mehr verpflichtet sein, sich laufend zu bewerben oder an Fördermaßnahmen teilzunehmen. Dies bedeutet allerdings meist keine generelle Absage an Erwerbstätigkeit: Ein Viertel der westdeutschen und $28 \%$ der ostdeutschen Nutzer bejahten zwar mindestens eines dieser beiden Items, wollten aber zugleich noch arbeiten und sahen sich dazu hinsichtlich ihrer Gesundheit und allgemeinen Leistungsfähigkeit auch noch in der Lage (Tab. 3). Von dieser Gruppe ist daher anzunehmen, dass sie gemäß der Autonomie-Hypothese den ELB vorrangig gewählt hat, um sich von den Zwängen einer aktivierenden Arbeitsmarktpolitik zu befreien.

Die vorgestellten Ergebnisse deuten darauf hin, dass in den meisten Fällen weniger der fehlende Wille, zu arbeiten, als vielmehr Entmutigung die Nutzung des ELB maßgeblich motiviert haben. ${ }^{12}$ Für diese Interpretation sprechen auch die Befunde aus derselben Befragung zum Suchverhalten und den Gründen für die Beendigung der Arbeitssuche: Erwartungsgemäß suchten zum Interviewzeitpunkt nur jeweils $17 \%$ der ost- und westdeutschen Nutzer des ELB tatsächlich noch aktiv nach einer Erwerbstätigkeit. Die übrigen Nutzer begründeten ihren Verzicht auf Suchaktivitäten

\footnotetext{
${ }^{11}$ Subjektiv empfundene Erwerbsunfähigkeit einerseits sowie fehlende Stellen- oder Förderangebote andererseits werden hier getrennt betrachtet, obwohl sie tatsächlich zwei Seiten desselben Problems darstellen: Beide Gründe für den unfreiwilligen Rückzug Älterer in den Vorruhestand beruhen auf der Einschätzung der Betroffenen, dass es auf dem Arbeitsmarkt keine Nachfrage nach Personen mit ihren Kompetenzen und Eigenschaften gibt und dass auch die Arbeitsvermittler das Problem der mangelnden Passung zwischen den persönlichen Ressourcen und den Anforderungen der verfügbaren Stellen nicht lösen können oder wollen (siehe dazu auch die Diskussion um die individuelle sowie die institutionelle und arbeitsnachfrageseitige Komponente der Beschäftigungsfähigkeit u.a. in Promberger et al. 2008 und McQuaid und Lindsay 2005). Für die Trennung der beiden Nutzertypen spricht jedoch, dass die Befragten selbst diesbezüglich differenziert haben und den Fokus entweder nur auf die arbeitsnachfrageseitigen und institutionellen Eingliederungshürden richteten oder (zusätzlich) die Probleme in ihrer Person begründet sahen.

${ }^{12} \mathrm{Zu}$ ähnlichen Resultaten gelangen auch Beste et al. (2010, S. 6), bezogen auf die Gründe von Arbeitslosengeld-II-Beziehern, aktuell nicht nach einer Arbeit zu suchen, obwohl sie dazu nach eigenen Angaben verpflichtet sind. Zudem decken sich die vorgestellten Befunde weitgehend mit den in britischen Studien erhobenen Auskünften von älteren Beziehern des Incapacity Benefit (Arbeitsunfähigkeitsgelds) und von anderen Gruppen älterer nicht erwerbstätiger Personen zu ihrer Erwerbsbereitschaft und ihren Erwerbsbarrieren (Beatty und Fothergill 2007: 70-73; Humphrey et al. 2003: 93-108; Riach und Loretto 2009: $108-110)$.
}

bei weitem am häufigsten mit der Einschätzung, aus Altersgründen nicht mehr vermittelbar zu sein. Dieses Motiv nannten $73 \%$ der westdeutschen und sogar $82 \%$ der ostdeutschen Nutzer. Im Vergleich dazu gaben z.B. nur $25 \%$ der Älteren in West- und $35 \%$ in Ostdeutschland als Grund an, dass der Arbeitsmarkt keine Stellen mit angemessener Entlohnung biete. ${ }^{13}$ Demnach ist die Nutzung des ELB in der Untersuchungsgruppe also eher als unfreiwillig akzeptierter denn als strategisch gewählter Vorruhestand zu interpretieren. Für die meisten Betroffenen dürfte sich die Wahl des ELB somit vorrangig als Entscheidung zwischen Langzeitarbeitslosigkeit und Vorruhestand und weniger als Arbeitsangebotsentscheidung zwischen Erwerbsarbeit und Freizeit dargestellt haben.

\subsection{Sozioökonomische Bestimmungsgründe der individuellen Entscheidungen}

Ergänzend zu den subjektiven Sichtweisen der Betroffenen geht es im Folgenden um die Frage, welche sozioökonomischen Faktoren die individuellen Entscheidungen für oder gegen den ELB beeinflusst haben. Dazu wurden zwei Modelle geschätzt: eine binäre logistische Regression, in der die Wahl oder Nichtwahl dieser Regelung den zu erklärenden Sachverhalt darstellt, sowie eine multinomiale logistische Regression, bei der die im letzten Abschnitt entwickelten vier „Nutzertypen“ (Tab. 3) und die Gruppe der Nichtnutzer als Referenzkategorie die abhängige Variable bilden. Die Auswahl der in beiden Modellen identischen unabhängigen Variablen stützt sich auf die umfangreiche Forschungsliteratur zu den Determinanten des Übergangs in den Ruhestand (z.B. Börsch-Supan 2000; Brussig und Stegmann 2007; Drobnič 2002; Ebbinghaus 2006; Radl 2007; Wübbeke 2005) sowie auf Untersuchungen zu den Lohnkonzessionen von Arbeitslosen (vor allem Bender et al. 2008): Erstens wurden Faktoren einbezogen, von denen angenommen wird, dass sie auf die Erwerbsneigung und Ruhestandsorientierung älterer Arbeitsloser und damit auf die Wahrscheinlichkeit eines freiwilligen Übergangs in den Vorruhestand eingewirkt haben. Dazu zählen die finanzielle Absicherung des Haushalts im Ruhestand, die Existenz eines Ehepartners als Indikator für den Wunsch nach Zeit für die Familie, der frühere berufliche Status als Indikator für die Berufsbindung, die Dauer der Ausgliederung aus regulärer Erwerbstätigkeit als Indikator für Gewöhnungsund Anpassungsprozesse und - nur in Westdeutschland -

\footnotetext{
${ }^{13}$ Interessanterweise begründeten zudem lediglich jeweils $23 \%$ der west- und ostdeutschen Nutzer ihre Nichtsuche damit, dass sie sich bereits im Ruhestand befänden. Dieses Ergebnis steht in Einklang mit den Befunden einer aktuellen britischen Studie auf Basis der „Sheffield Hallam surveys" (Beatty und Fothergill 2007, S. 70-73) sowie mit älteren Studien aus Frankreich und Großbritannien (siehe dazu Henkens et al. 1996, S. 563).
} 
das Geschlecht. ${ }^{14}$ Zweitens umfassen die Modelle Faktoren, welche die Neigung beeinflusst haben dürften, den ELB aus dem Autonomiemotiv heraus zu wählen. Diese Neigung sollte für diejenigen Personen geringer gewesen sein, die unter einem hohen ökonomischen Druck standen, wieder Arbeit zu finden, oder deren individuelle Arbeitsmarktchancen besonders ungünstig waren: Von diesen Personen ist anzunehmen, dass sie eher bereit waren, die an den Arbeitslosenstatus gebundenen Pflichten, insbesondere die Pflicht zur Annahme jedes zumutbaren Arbeitsangebotes, in Kauf zu nehmen, um weiterhin Zugang zu den Dienst- und Förderleistungen der Arbeitsvermittlung zu haben. $\mathrm{Zu}$ den Variablen, welche Einfluss auf das Streben nach Autonomie gehabt haben dürften, zählen zusätzlich zu den oben bereits erwähnten Merkmalen die individuellen Deutschkenntnisse und die regionale Arbeitsmarktlage (gemessen anhand des Wohnorts in West- oder Ostdeutschland, denn im betrachteten Zeitraum war die Arbeitslosenquote in den neuen Ländern rund doppelt so hoch wie in den alten). Drittens beinhalten die Modelle Faktoren, die das Risiko eines unfreiwilligen, durch subjektive Erwerbsunfähigkeit begründeten Wechsels in den Vorruhestand mitbestimmt haben dürften. Dazu gehören das Vorliegen einer Behinderung oder Schwerbehinderung, die Ausgliederungsdauer als Indikator für die Erosion von Qualifikationen und Selbstvertrauen sowie die frühere berufliche Stellung als Indikator für bisherige Arbeitsbelastungen und das Qualifikationsniveau. Die vierte Gruppe von Variablen schließlich umfasst Einflussgrößen der Wahrscheinlichkeit, sich aufgrund fehlender Stellen- und Förderangebote für den ELB zu entscheiden. Hier dürften erneut die regionale Arbeitsmarktlage, die frühere Stellung im Beruf, die Ausgliederungsdauer und die Deutschkenntnisse eine Rolle gespielt haben, aber auch das Geschlecht, da Frauen im Beobachtungszeitraum an den meisten Maßnahmen der aktiven Arbeitsmarktpolitik nur unterproportional partizipierten (Bundesagentur für Arbeit 2007, S. 123 f.; Heinemann et al. 2006, S. 32 f.).

Als Kontrollvariablen wurden zusätzlich die Zeitdauer seit Entstehung des persönlichen Anspruchs auf den ELB,

\footnotetext{
${ }^{14}$ In die Modelle wurde das Geschlecht nicht als separates Merkmal einbezogen, sondern mit dem Merkmal „Wohnort in West- oder Ostdeutschland" zu einer Variablen kombiniert. Damit soll berücksichtigt werden, dass ostdeutsche Frauen und Männer der betrachteten Generation aufgrund der spezifischen wirtschaftlichen und gesellschaftlichen Rahmenbedingungen in der Deutschen Demokratischen Republik während des Großteils ihres Erwerbslebens relativ gleichberechtigt am Arbeitsleben beteiligt waren. Frauen und Männer in den neuen Bundesländern dürften sich deshalb hinsichtlich ihres Rollenverständnisses, der bisherigen Bedeutung ihrer Berufstätigkeit für das Haushaltseinkommen sowie der Erwerbsneigung und Berufsbindung ähnlicher sein als die gleichaltrigen Frauen und Männer in den alten Bundesländern. Dort dominiert in der untersuchten Generation noch das Modell des männlichen Familienernährers, so dass die Erwerbsorientierung der westdeutschen Frauen im Allgemeinen schwächer sein dürfte als die der westdeutschen Männer.
}

das Alter zu Beginn dieses Zeitraums sowie die Art der Leistung, die der ältere Arbeitslose vor dem Wechsel in das Arbeitslosengeld II bezogen hatte, berücksichtigt. ${ }^{15}$

Blickt man zunächst auf die Ergebnisse des binären Modells (Tab. $4,{ }^{16}$ Sp. 4), so wird deutlich, dass kein anderer Faktor den Rückzug älterer Arbeitsloser stärker förderte als eine positive Einschätzung der finanziellen Absicherung im Rentenalter: Befragte, die mit einer deutlichen Verbesserung ihrer Einkommenslage nach Eintritt in den Ruhestand rechneten, und die Besitzer von schuldenfreiem Wohneigentum optierten jeweils deutlich häufiger für den ELB als die schlechter abgesicherten Befragten. Darüber hinaus neigten Verheiratete, ostdeutsche Frauen und Arbeitslose, die bereits mindestens fünf Jahre lang nicht mehr regulär erwerbstätig waren, signifikant stärker zur Wahl des ELB. Demgegenüber nahmen Personen mit mittelmäßigen oder schlechten Deutschkenntnissen diese Regelung weitaus seltener in Anspruch als ältere Arbeitslose mit guten oder sehr guten deutschen Sprachkenntnissen.

Welche Handlungsrationalitäten diesen Effekten jeweils zugrunde liegen, zeigen die Resultate des multinomialen Modells: Demnach erhöhte die Erwartung einer besseren materiellen Lage im Ruhestand signifikant die Neigung, aufgrund fehlender Arbeitswilligkeit für den ELB zu optieren (Tab. 4, Sp. 5). Dies entspricht den Erwartungen des mikroökonomischen Erklärungsansatzes und deutet darauf hin, dass ein Teil der Betroffenen die Wahl oder Nichtwahl des ELB tatsächlich bis zu einem gewissen Grad als eine

\footnotetext{
${ }^{15}$ Die Zeitdauer seit Entstehung des persönlichen Anspruchs auf den ELB kontrolliert für die Tatsache, dass ein Teil der Befragten zum Interviewzeitpunkt erst seit Kurzem die Möglichkeit hatte, den ELB zu nutzen, und daher möglicherweise über diese Option noch nicht informiert war oder diesbezüglich noch keine Entscheidung getroffen hatte. Die Variable „Alter“ dient der Kontrolle eines Selektionseffektes: Da Personen unter bestimmten Bedingungen bereits ab dem vollendeten 60. Lebensjahr eine Altersrente beziehen konnten, ist zu erwarten, dass mit zunehmendem Alter der Anteil von arbeitsbereiten Personen im Leistungsbezug wuchs, weil ruhestandsorientierte Personen in Rente wechselten. Erwartungsgemäß zeigen die Schätzungen, dass Personen ab dem Alter von 62 Jahren signifikant seltener für den ELB optiert haben. Die Variable zur Art des Leistungsbezugs vor dem Übergang in das Arbeitslosengeld II schließlich kontrolliert für die vielfältigen sozialen Unterschiede zwischen früheren Sozialhilfebeziehern einerseits und ehemaligen Arbeitslosenhilfebeziehern (sowie Beziehern anderer Entgeltersatzleistungen) andererseits (siehe Abschn. 3.1 „Daten und Untersuchungspopulation“).

${ }^{16}$ Tabelle 4 gibt die Ergebnisse des binären Modells als Odds-Ratios und die Resultate des multinomialen Modells als Conditional-OddsRatios wieder. Ein Odds-Ratio größer als 1 zeigt an, dass das betreffende Merkmal die Neigung zur Inanspruchnahme des ELB im Vergleich zur jeweiligen Referenzkategorie der unabhängigen Variable erhöht hat, während Werte kleiner als 1 umgekehrt einen negativen $\mathrm{Zu}$ sammenhang signalisieren. Analog dazu besagt ein Conditional-OddsRatio größer oder kleiner als 1, dass das Merkmal die Chance, dem betreffenden Nutzertyp und nicht der Gruppe der Nichtnutzer anzugehören, im Vergleich zur jeweiligen Referenzkategorie vergrößert oder verringert hat.
} 
Tab. 4 Binäre und multionomiale logistische Regression zu den Determinanten der Inanspruchnahme des ELB

\begin{tabular}{|c|c|c|c|c|c|c|c|}
\hline & \multirow[t]{3}{*}{$n$} & \multirow[t]{3}{*}{$\%$} & \multirow{3}{*}{$\begin{array}{l}\text { Modell } 1 \\
\text { Odds-Ratios } \\
\text { Inan- } \\
\text { spruch- } \\
\text { nahme } \\
\text { des ELB }\end{array}$} & \multicolumn{4}{|c|}{$\begin{array}{l}\text { Modell } 2 \\
\text { Conditional Odds-Ratios }\end{array}$} \\
\hline & & & & \multicolumn{4}{|c|}{ Motivation für die Nutzung des ELB } \\
\hline & & & & $\begin{array}{l}\text { Wollte } \\
\text { nicht } \\
\text { mehr } \\
\text { arbeiten }\end{array}$ & $\begin{array}{l}\text { Wollte } \\
\text { autonom } \\
\text { sein }\end{array}$ & $\begin{array}{l}\text { Konnte } \\
\text { nicht } \\
\text { mehr } \\
\text { arbeiten }\end{array}$ & $\begin{array}{l}\text { Habe } \\
\text { keine } \\
\text { Hilfe } \\
\text { erhalten }\end{array}$ \\
\hline \multicolumn{8}{|l|}{ Wohnort und Geschlecht } \\
\hline Mann aus Westdeutschland & 305 & 34 & 1 & 1 & 1 & 1 & 1 \\
\hline Frau aus Westdeutschland & 187 & 21 & 1,09 & 1,82 & $0,53^{*}$ & 1,17 & 1,22 \\
\hline Mann aus Ostdeutschland & 222 & 25 & 1,14 & 1,33 & 0,95 & 0,87 & $1,75^{*}$ \\
\hline Frau aus Ostdeutschland & 175 & 20 & $2,03^{* * *}$ & 1,44 & 1,52 & $1,87^{* *}$ & $3,09^{* * *}$ \\
\hline \multicolumn{8}{|l|}{ Familienstand } \\
\hline verheiratet & 475 & 53 & $1,32^{*}$ & 1,03 & 1,38 & 1,28 & 1,39 \\
\hline nicht verheiratet & 414 & 47 & 1 & 1 & 1 & 1 & 1 \\
\hline \multicolumn{8}{|l|}{ Deutschkenntnisse } \\
\hline (sehr) gut & 714 & 80 & 1 & 1 & 1 & 1 & 1 \\
\hline mittelmäßig oder schlecht & 175 & 20 & $0,35^{* * *}$ & $0,33^{* *}$ & $0,24^{* * *}$ & $0,47^{* * *}$ & $0,32^{* * *}$ \\
\hline \multicolumn{8}{|l|}{ Gesundheitliche Beeinträchtigungen } \\
\hline nicht behindert & 681 & 77 & 1 & 1 & 1 & 1 & 1 \\
\hline behindert & 118 & 13 & 1,06 & 0,80 & $0,30^{* * *}$ & $2,90^{* * *}$ & $0,45^{* *}$ \\
\hline schwerbehindert & 90 & 10 & 0,72 & 0,60 & $0,23^{* * *}$ & $1,81^{* *}$ & $0,24^{* * *}$ \\
\hline \multicolumn{8}{|c|}{ Letzte Stellung im Beruf vor Eintritt in den Leistungsbezug } \\
\hline $\begin{array}{l}\text { niedrige berufliche Stellung (angelernte } \\
\text { Arbeiter, Hilfsarbeiter, Angestellte mit } \\
\text { einfacher Tätigkeit) }\end{array}$ & 258 & 29 & 0,86 & 1,05 & 0,71 & 0,88 & 0,88 \\
\hline $\begin{array}{l}\text { mittlere berufliche Stellung (Facharbeiter, } \\
\text { Gesellen, Gehilfen, Vorarbeiter, } \\
\text { Kolonnenführer, Brigadenführer, } \\
\text { Angestellte mit qualifizierter Tätigkeit) }\end{array}$ & 281 & 32 & 1 & 1 & 1 & 1 & 1 \\
\hline $\begin{array}{l}\text { hohe berufliche Stellung (Meister, Poliere } \\
\text { im Arbeiterverhältnis, Angestellte mit } \\
\text { hochqualifizierter Tätigkeit) }\end{array}$ & 79 & 9 & 0,96 & 0,70 & 1,00 & 1,07 & 0,91 \\
\hline $\begin{array}{l}\text { selbständig (selbstständige Landwirte, } \\
\text { freie Berufe, selbstständige Akademiker, } \\
\text { sonstige Selbstständige, freie Mitarbeiter } \\
\text { und mithelfende Familienangehörige) und } \\
\text { unbekannte Stellung }\end{array}$ & 271 & 30 & 0,79 & $0,37^{* *}$ & 0,73 & 0,96 & 0,87 \\
\hline \multicolumn{8}{|c|}{ Dauer seit Ende der letzten (mehr als geringfügigen) Erwerbstätigkeit zu Beginn des Anspruchs auf ELB } \\
\hline unter $1 \mathrm{Jahr}$ & 169 & 19 & 1 & 1 & 1 & 1 & 1 \\
\hline 1 - unter 2 Jahre & 78 & 9 & 0,77 & 0,42 & 0,84 & 0,98 & 0,59 \\
\hline 2 - unter 5 Jahre & 244 & 27 & 1,39 & 1,11 & 1,02 & $1,81^{*}$ & 1,42 \\
\hline 5 oder mehr Jahre & 398 & 45 & $1,50^{*}$ & $2,30^{*}$ & 1,04 & $1,72^{*}$ & 1,44 \\
\hline \multicolumn{8}{|c|}{ Art des Leistungsbezugs vor Einführung des Arbeitslosengelds II } \\
\hline Sozialhilfe & 125 & 14 & $0,38^{* * *}$ & $0,17^{* *}$ & $0,36^{*}$ & 0,56 & $0,22^{* * *}$ \\
\hline $\begin{array}{l}\text { Arbeitslosenhilfe, Arbeitslosengeld, } \\
\text { sonstige oder keine Leistung }\end{array}$ & 764 & 86 & 1 & 1 & 1 & 1 & 1 \\
\hline \multicolumn{8}{|l|}{ Alter zu Beginn des Anspruchs auf ELB } \\
\hline 58 Jahre & 678 & 76 & 1 & 1 & 1 & 1 & 1 \\
\hline 59 Jahre & 72 & 8 & $0,61^{*}$ & 0,71 & 0,72 & 0,75 & $0,28^{* *}$ \\
\hline 60 Jahre & 49 & 6 & 0,75 & 1,41 & 0,55 & 0,70 & 0,74 \\
\hline 61 Jahre & 42 & 5 & 0,87 & 2,44 & 0,68 & 0,84 & 0,70 \\
\hline 62 Jahre oder älter & 48 & 5 & $0,27^{* * *}$ & 0,38 & $0,26^{*}$ & $0,14^{* *}$ & 0,43 \\
\hline
\end{tabular}


Tab. 4 (Fortsetzung)

\begin{tabular}{|c|c|c|c|c|c|c|c|}
\hline & \multirow[t]{3}{*}{$n$} & \multirow[t]{3}{*}{$\%$} & \multirow{3}{*}{$\begin{array}{l}\text { Modell } 1 \\
\text { Odds-Ratios } \\
\text { Inan- } \\
\text { spruch- } \\
\text { nahme } \\
\text { des ELB }\end{array}$} & \multicolumn{4}{|c|}{$\begin{array}{l}\text { Modell } 2 \\
\text { Conditional Odds-Ratios }\end{array}$} \\
\hline & & & & \multicolumn{4}{|c|}{ Motivation für die Nutzung des ELB } \\
\hline & & & & $\begin{array}{l}\text { Wollte } \\
\text { nicht } \\
\text { mehr } \\
\text { arbeiten }\end{array}$ & $\begin{array}{l}\text { Wollte } \\
\text { autonom } \\
\text { sein }\end{array}$ & $\begin{array}{l}\text { Konnte } \\
\text { nicht } \\
\text { mehr } \\
\text { arbeiten }\end{array}$ & $\begin{array}{l}\text { Habe } \\
\text { keine } \\
\text { Hilfe } \\
\text { erhalten }\end{array}$ \\
\hline \multicolumn{3}{|l|}{$\begin{array}{l}\text { Zeitdauer seit Entstehung des } \\
\text { persönlichen Anspruchs auf ELB in } \\
\text { Monaten }\end{array}$} & $1,06^{* * *}$ & 1,04 & $1,09^{* * *}$ & $1,04^{* *}$ & $1,07^{* * *}$ \\
\hline \multicolumn{3}{|l|}{$\begin{array}{l}\text { Zeitdauer seit Entstehung des } \\
\text { persönlichen Anspruchs auf ELB } \\
\text { quadriert }\end{array}$} & $1,00^{* * *}$ & 1,00 & $1,00^{* * *}$ & $1,00^{*}$ & $1,00^{* *}$ \\
\hline \multicolumn{8}{|c|}{ Einschätzung der Einkommenslage im Ruhestand (eigenes und Partnereinkommen) im Vergleich zum aktuellen Einkommen } \\
\hline Einkommen im Ruhestand niedriger & 286 & 32 & 0,94 & 0,47 & 1,11 & 1,08 & 0,94 \\
\hline $\begin{array}{l}\text { Einkommen im Ruhestand etwa gleich } \\
\text { hoch }\end{array}$ & 158 & 18 & 1 & 1 & 1 & 1 & 1 \\
\hline Einkommen im Ruhestand etwas höher & 289 & 33 & 1,40 & 1,25 & 1,68 & 1,50 & 1,19 \\
\hline $\begin{array}{l}\text { Einkommen im Ruhestand erheblich } \\
\text { höher }\end{array}$ & 82 & 9 & $2,19^{* *}$ & $2,66^{*}$ & $3,83^{* * *}$ & 1,24 & $2,15^{*}$ \\
\hline $\begin{array}{l}\text { keine Einschätzung zum Einkommen im } \\
\text { Ruhestand }\end{array}$ & 74 & 8 & 0,78 & 0,45 & $0,23^{*}$ & 1,16 & 0,99 \\
\hline \multicolumn{8}{|l|}{ Wohneigentum } \\
\hline keins & 709 & 80 & 1 & 1 & 1 & 1 & 1 \\
\hline mit Schulden belastet & 90 & 10 & 0,85 & 1,41 & 0,66 & 0,66 & 1,11 \\
\hline schuldenfrei & 90 & 10 & $1,82^{* *}$ & 1,03 & $1,87^{*}$ & 1,59 & $2,44^{* *}$ \\
\hline Fallzahl & 889 & 100 & 518 & 52 & 139 & 171 & 156 \\
\hline \multicolumn{3}{|l|}{ McFaddens Pseudo-R-Quadrat } & 0,18 & & & 0,15 & \\
\hline \multicolumn{3}{|l|}{ Adjustet Count R Quadrat } & 0,35 & & & 0,15 & \\
\hline \multicolumn{8}{|c|}{ Signifikanzniveau: ${ }^{*} p<0,10 ;{ }^{* *} p<0,05 ;{ }^{* * *} p<0,01$} \\
\hline
\end{tabular}

Quelle: IAB-Querschnittbefragung „Lebenssituation und Soziale Sicherung 2005“(QS I); ungewichtet; eigene Berechnungen

Hinweis: Referenzkategorie der abhängigen Variable ist in beiden Modellen die Entscheidung, den ELB nicht zu nutzen

ökonomische Arbeitsangebotsentscheidung traf. ${ }^{17}$ Auch eine Ausgliederungsdauer von mindestens fünf Jahren ließ es wahrscheinlicher werden, dass sich Ältere zur Nutzung des ELB entschlossen, weil sie keine Arbeit mehr aufnehmen wollten. Dieser Befund lässt sich ebenfalls im Sinne des mikroökonomischen Arbeitsangebotsmodells interpretieren. So könnte der Effekt der Ausgliederungsdauer Folge einer zunehmenden Entfremdung vom Erwerbsprozess, einer Gewöhnung an das Leben ohne Arbeit und eines sinkenden individuell erreichbaren Marktlohns sein und daher mit der im Zeitverlauf zunehmenden Attraktivität von Freizeit erklärt werden. Aus psychologischer Perspektive könnte

\footnotetext{
${ }^{17}$ Dieses Ergebnis stimmt mit dem Befund von P.R. Jackson und P.E. Taylor (1994, S. 559, 563) überein, wonach finanzieller Druck ältere männliche Arbeitslose daran hindert, sich vom Arbeitsmarkt zurückzuziehen und in die psychisch entlastende Rolle des Ruheständlers zu wechseln, während das Gefühl finanzieller Sicherheit die Loslösung von der Erwerbsrolle erleichtert.
}

sich in diesem Effekt aber ebenso das Bemühen betroffener Arbeitsloser widerspiegeln, die persönlichen Ziele mit den sich verschlechternden Beschäftigungschancen in Einklang zu bringen und damit motivationale Inkongruenz zu beseitigen („Ich will ja auch gar nicht mehr arbeiten“).

Im Gegensatz dazu zeigten Personen, die ehemals selbständig tätig waren oder deren frühere berufliche Stellung nicht bekannt ist, eine besonders geringe Neigung, sich aufgrund des fehlenden Willens zur Arbeit für den Vorruhestand zu entscheiden. Dies dürfte auf eine vergleichsweise starke Erwerbsbereitschaft der früheren Selbständigen innerhalb dieser Gruppe zurückzuführen sein: Da die berufliche Selbständigkeit typischerweise hohen Arbeitseinsatz verlangt und zugleich mit einem tendenziell hohen Einkommen und Sozialprestige verbunden ist, ist anzunehmen, dass sich diese Personen in besonderem Maße mit ihrem Beruf und ihrer Erwerbsarbeit identifizierten. Ein weiterer Grund könnte in der häufig unzureichenden Altersvorsorge von 
Selbständigen liegen, deren Effekt durch die entsprechenden Indikatoren möglicherweise nicht vollständig aufgefangen wurde.

Positiv auf die Wahrscheinlichkeit, den ELB aus dem Autonomie-Motiv zu nutzen (Tab. 4, Sp. 6), wirkte ausschließlich eine relativ gute Absicherung im Alter. Offenbar waren Personen, die mit einem höheren Haushaltseinkommen im Ruhestand rechneten, sowie Ältere mit schuldenfreiem Wohneigentum seltener dazu bereit, sich den Zwängen der aktivierenden Arbeitsmarktpolitik auszusetzen und insbesondere Zugeständnisse bei den Ansprüchen an eine neue Stelle zu machen, als Ältere, die aufgrund unzureichender Altersvorsorge unter einem höheren ökonomischen Druck standen, nochmals eine Arbeit aufzunehmen.

Eine besonders schwache Neigung, den ELB aus dem Autonomie-Motiv heraus zu wählen, zeigten hingegen westdeutsche Frauen, Personen, die keine Einschätzung zu ihrem späteren Ruhestandseinkommen abgeben wollten oder konnten, sowie Behinderte und Schwerbehinderte. Letzteres ist ein Indiz dafür, dass sich ältere Erwerbslose dann seltener der Aktivierung entziehen, wenn sie im Gegenzug von der betreuenden Stelle wirksame Hilfe und Förderung erwarten können. Denn für die berufliche Reintegration von Arbeitslosen mit starken gesundheitlichen Einschränkungen stehen in Deutschland besondere Förderinstrumente zur Verfügung. Darüber hinaus könnte die höhere Konzessionsbereitschaft dieser Gruppe auf ihren tendenziell schlechteren Arbeitsmarktchancen sowie einer überdurchschnittlich hohen Erwerbsorientierung beruhen: Personen mit gesundheitlichen Handicaps könnten wegen ihrer doppelten Diskriminierung als Behinderte und Arbeitslose besonders stark an einer Reintegration in Beschäftigung interessiert sein, um ihre soziale Integration zu verbessern und ihr Selbstwertgefühl zu stärken.

Zugleich optierten Behinderte und Schwerbehinderte jedoch signifikant häufiger für den ELB, weil sie sich trotz grundsätzlicher Arbeitswilligkeit den beruflichen Anforderungen aus gesundheitlichen oder anderen Gründen nicht mehr gewachsen fühlten (Tab. 4, Sp. 7). Dasselbe gilt für Personen mit einer Ausgliederungsdauer von mindestens zwei Jahren sowie für ostdeutsche Frauen. Letztere wechselten zudem bei weitem öfter als westdeutsche Frauen und Männer und auch häufiger als Männer aus Ostdeutschland wegen fehlender Stellen- und Förderangebote in den ELB (Tab. 4, Sp. 8). So waren die Chancen (Odds) ostdeutscher Frauen, diese Regelung als Reaktion auf fehlende Unterstützung zu wählen, dreifach so hoch wie die westdeutscher Männer (Referenzkategorie) und damit einer der stärksten positiven Effekte überhaupt. Der Rückzug ostdeutscher Frauen in den Vorruhestand ist somit in erster Linie ihrer Resignation geschuldet: Unter ihnen war das Gefühl der eigenen Chancenlosigkeit auf dem Arbeitsmarkt offenbar besonders stark verbreitet und sie erwarteten auch von der öffentlichen Arbeitsvermittlung häufiger als die übrigen Älteren keine wirksame Hilfe. Ein ähnliches Bild zeigt sich auch für ostdeutsche Männer, die vor dem Hintergrund der schlechten regionalen Arbeitsmarktlage ebenfalls deutlich öfter als westdeutsche Betroffene aufgrund fehlender Stellen- und Maßnahmeangebote den Vorruhestand wählten. Entgegen den Erwartungen hatten zudem Ältere, die sich im Hinblick auf die Ruhestandsphase relativ gut abgesichert fühlten, höhere Chancen, zu diesem Nutzertyp und nicht zu den Nichtnutzern zu gehören, als Personen mit schlechterer Altersvorsorgelage.

Umgekehrt war die Wahrscheinlichkeit, wegen fehlender institutioneller Hilfe für den Ausstieg zu optieren, besonders niedrig bei Behinderten und insbesondere Schwerbehinderten. Dies steht in Einklang mit den weiter oben dargestellten Beobachtungen und weist darauf hin, dass ältere Erwerbslose mit gesundheitlichen Beeinträchtigungen tendenziell besser gefördert wurden oder zumindest eine bessere Förderung erwartet haben als Ältere ohne diese Handicaps.

Eine weitere Personengruppe scheint den ELB vor allem aufgrund von Informationsdefiziten besonders wenig genutzt zu haben. Dies betrifft die Personen mit schlechten oder mittelmäßigen deutschen Sprachkenntnissen. So verringerten Sprachprobleme die Wahrscheinlichkeit der $\mathrm{Zu}$ gehörigkeit zu jeder der vier Nutzergruppen gleichermaßen stark. Daher liegt die Vermutung nahe, dass Personen mit schlechten Deutschkenntnissen von Arbeitsagenturen und Grundsicherungsstellen häufig nicht in für sie verständlicher Weise über diese Regelung aufgeklärt wurden, möglicherweise, weil fremdsprachiges Informationsmaterial fehlte. Trifft diese Interpretation zu, spiegelt sich in diesem Befund die große Bedeutung der Informations- und Beratungspolitik der Träger für die Steuerung der Inanspruchnahme des ELB wider. Der Migrationshintergrund selbst hatte bei Kontrolle der Deutschkenntnisse dagegen keinen signifikanten Effekt auf die Nutzung des ELB, wie zusätzliche Modellschätzungen zeigten (Brussig und Wübbeke 2009, S. 271 und 273).

\section{Schlussbemerkungen}

Der ELB setzte für die Gruppe der älteren Erwerbslosen ein Grundprinzip aktivierender Arbeitsmarktpolitik außer Kraft: Er garantierte den Betroffenen finanzielle Leistungen bis zum Rentenbeginn, ohne sie zur Arbeitssuche und zur Annahme zumutbarer Stellenangebote zu verpflichten. Diese Regelung spiegelt die Spannungen und Widersprüche zwischen politischen Zielen und sozialen Realitäten in Bezug auf ältere Arbeitnehmer wider: Einerseits soll der Vorruhestand zurückgedrängt werden, um das wachsende $\mathrm{Ar}$ beitskräftepotenzial der Älteren besser zu nutzen, die Sozialsysteme finanziell zu stabilisieren und Altersarmut so- 
wie sozialer Exklusion entgegenzuwirken; darauf sind zahlreiche Maßnahmen der jüngeren Arbeitsmarkt- und Sozialreformen ausgerichtet. Andererseits stößt die berufliche Reintegration älterer Beschäftigungsloser aber auf beträchtliche Probleme, sodass ihre Aktivierung unter betriebswirtschaftlichen Effizienzgesichtspunkten häufig als nicht lohnend erscheint, während ihr vorzeitiger Ruhestand umgekehrt die Arbeitslosenstatistik und Arbeitsvermittlung unmittelbar entlastet. Hinzu kommt, dass Möglichkeiten zum vorzeitigen Ausstieg aus dem Arbeitsleben bei den Wählern sehr populär sind. Die Vorzüge von Vorruhestandsregelungen für Politik und Arbeitsverwaltung sind somit offenkundig. Weniger klar ist hingegen, was die älteren Arbeitslosen selbst dazu veranlasst, sich vom Arbeitsmarkt zurückzuziehen, obwohl vertiefte Erkenntnisse darüber Anhaltspunkte zur Verbesserung der Arbeitsmarktpolitik für diese Gruppe geben könnten. Der vorliegende Beitrag widmete sich dieser Frage, indem er auf Basis von Befragungsdaten untersuchte, aus welchen Gründen sich die Mehrheit der ab 58-jährigen Arbeitslosengeld-II-Bezieher in Deutschland für die Inanspruchnahme des ELB entschieden hatte: Wechselten sie vorrangig aufgrund mangelnder Arbeitswilligkeit in den Ruhestand, wie mikroökonomische Arbeitsangebotstheorien erwarten lassen, oder aus Resignation angesichts fehlender Beschäftigungsaussichten, worauf Studien aus der soziologisch-psychologischen Arbeitslosigkeitsforschung hinweisen?

Die Aussagekraft der hier präsentierten Analysen unterliegt dabei einer Einschränkung: Da die Gründe für die Wahl des ELB retrospektiv erhoben worden sind, können nachträgliche Rationalisierungsversuche die tatsächlichen Handlungsmotive bei einem Teil der Befragten verdeckt haben. Dabei ist angesichts der sensitiven Fragen nach Erwerbsbereitschaft und Arbeitswilligkeit insbesondere mit Verzerrungen durch sozial erwünschte Antworten zu rechnen. Die Untersuchung des Arbeitsmarktverhaltens älterer Arbeitsloser anhand der Regelung des ELB scheint aber ein möglicher Weg zu sein, um solche Verzerrungen zumindest zu begrenzen, da die Bereitschaft, fehlende Arbeitswilligkeit im Interview offenzulegen, bei denjenigen älteren Leistungsempfängern höher sein dürfte, denen der Gesetzgeber explizit das Recht zugebilligt hat, nicht mehr arbeiten zu wollen. Ergänzende Auswertungen stützten diese Vermutung, so dass davon ausgegangen wird, dass die Angaben der Befragten wenigstens in der Tendenz ein zutreffendes Bild von den Gründen für ihre Abkehr vom Arbeitsmarkt zeichnen.

Die von den Befragten berichteten Motive für die Wahl des ELB zeigen, dass der Rückzug älterer Langzeitarbeitsloser aus der Aktivierung und Vermittlung nicht in erster Linie in der mangelnden Erwerbsmotivation der Betroffenen gründet: Nur rund ein Achtel der befragten Nutzer wählte die Ausstiegsoption, weil sie tatsächlich nicht mehr arbeiten wollte, und für ein weiteres Viertel der Nutzer hat- te die Erwerbs- und Mitwirkungsbereitschaft klare Grenzen: Sie optierten für den ELB hauptsächlich deshalb, weil sie nicht jede zumutbare Arbeit annehmen, kontinuierlich Bewerbungen nachweisen oder an angebotenen Fördermaßnahmen teilnehmen wollten. Die meisten der befragten Älteren hingegen wollten ihrem Selbstverständnis nach wieder in das Arbeitsleben zurückkehren, insbesondere dann, wenn sie noch keine ausreichende Absicherung für das Alter erreicht hatten, wie die Ergebnisse der multivariaten Analyse belegen. Der vielfache Wechsel älterer Erwerbsloser in den Vorruhestand ist daher nicht in erster Linie das Ergebnis nutzenmaximierender Entscheidungen zugunsten von Freizeit und gegen Erwerbsarbeit gewesen. Er kann deshalb auch nicht vorrangig als Problem falscher sozialrechtlicher Anreizwirkungen und insbesondere der negativen Arbeitsanreize des ELB selbst angesehen werden. Stattdessen scheint der wichtigste Grund für den Ausstieg der Älteren in den fehlenden Erwerbsperspektiven der Betroffenen zu liegen: Für mehr als $60 \%$ der Nutzer war die Einschätzung ausschlaggebend, den beruflichen Anforderungen aus gesundheitlichen oder anderen Gründen nicht mehr gewachsen zu sein, auf dem Arbeitsmarkt keine Chancen mehr zu haben und auch von der betreuenden Stelle keine ausreichende Hilfe und Förderung zu erhalten. Insbesondere für Männer aus Ostdeutschland, allen voran aber für ostdeutsche Frauen waren die Perspektivlosigkeit auf dem Arbeitsmarkt und geringe Erwartungen an die Unterstützungsbereitschaft und -möglichkeiten der Arbeitsverwaltung der dominierende Rückzugsgrund. Für die wirksame Aktivierung und Integration älterer Langzeiterwerbsloser dürfte es daher weniger auf ein strikteres „Fordern“ als vielmehr auf ein besseres „Fördern“ und geeignete Beschäftigungsmöglichkeiten ankommen. Besonderer Handlungsbedarf besteht dabei insbesondere bei der Förderung von Frauen; so korrespondiert die weit verbreitete Resignation unter ostdeutschen Hilfebezieherinnen mit der unterproportionalen Partizipation älterer Arbeitslosengeld-II-Bezieherinnen an Eingliederungsleistungen (Betzelt 2007, S. 301 f.; Boockmann et al. 2010, S. 285-290).

Vor dem Hintergrund der dargestellten Forschungsergebnisse ist die Abschaffung des ELB Ende 2007 grundsätzlich als positiv zu bewerten. So könnte dieser Schritt von den älteren Arbeitslosen als Signal verstanden werden, dass Politik und Arbeitsverwaltung sie nicht mehr als ,hoffnungslose Fälle" abschreiben, sondern sie als wichtigen Teil der Erwerbsbevölkerung betrachten, deren Integration in den Arbeitsmarkt ernsthaft angestrebt wird. Dasselbe Signal sendet auch der 2008 gesetzlich verankerte Auftrag an die Grundsicherungsstellen aus, erwerbsfähige Leistungsberechtigte, die das 58. Lebensjahr vollendet haben, unverzüglich in Arbeit oder in eine Arbeitsgelegenheit zu vermitteln (Paragraph 3 Absatz 2a SGB II). Über diese symbolische Wirkung hinaus können die gesetzlichen 
Änderungen aber nur dann zu einer tatsächlichen Verbesserung der Arbeitsmarkt- und gesellschaftlichen Teilhabechancen Älterer führen, wenn dem Ziel der Aktivierung und Integration dieser Gruppe im Konfliktfall Vorrang vor einer auf betriebswirtschaftliche Effizienz ausgerichteten Arbeitsmarktpolitik eingeräumt wird. ${ }^{18}$ Dies entspräche nicht nur einer konsistenten Politik der Lebensarbeitszeitverlängerung, sondern erscheint auch angesichts der deutlich verschlechterten sozialen Absicherung von älteren Arbeitslosen als geboten (siehe dazu Mika und Baumann 2008). Aktuelle Zahlen zur aktiven Arbeitsförderung der Älteren in den beiden Rechtskreisen SGB III und SGB II sprechen jedoch eher gegen eine solche Vorrangigkeit. So partizipieren ab 50-jährige Arbeitslose in der Regelförderung nach wie vor unterdurchschnittlich vor allem an qualifizierenden Maßnahmen, obwohl gerade in dieser Gruppe Qualifikationsdefizite ein wichtiges Vermittlungshemmnis darstellen und sich die Förderung der beruflichen Weiterbildung für ältere Arbeitslosengeld-II-Bezieher als besonders wirksam erwiesen hat (Deeke et al. 2011, S. 198). Je älter ältere Leistungsbezieher sind, desto seltener werden sie zudem gefördert; ab dem Alter von 60 Jahren sind Ältere von Maßnahmen der aktiven Arbeitsmarktpolitik sogar nahezu ausgeschlossen (Mümken et al. 2011, S. 57-59). Hinzu kommt, dass die jüngsten Sparvorgaben für die aktive Arbeitsmarktpolitik bereits zu deutlichen Kürzungen bei den Förderleistungen (nicht nur, aber auch) für ältere Erwerbslose geführt haben und in den nächsten Jahren weiter führen werden (Statistik der Bundesagentur für Arbeit 2011, S. 37-40). Auch die im Rahmen der Instrumentenreform vorgesehenen Restriktionen bei der öffentlich geförderten Beschäftigung dürften die Erwerbs- und Teilhabechancen von älteren Arbeitslosen mit schweren und multiplen Integrationshemmnissen zusätzlich beeinträchtigen.

Die Ambivalenz der Arbeitsmarktpolitik für Ältere zeigt sich zudem in zwei weiteren gesetzlichen Neuregelungen aus dem Jahr 2008, die das Ziel einer verbesserten Arbeitsmarktintegration älterer Langzeitarbeitsloser eher konterkarieren: Zum einen gelten Erwerbslose nun automatisch nicht mehr als arbeitslos, wenn sie nach ihrem 58. Geburtstag mindestens ein Jahr lang Leistungen der Grundsicherung für Arbeitsuchende bezogen haben, ohne dass ihnen eine sozialversicherungspflichtige Arbeitsstelle angeboten worden ist (Paragraph 53a Absatz 2 SGB II). Dies setzt Anreize für die Grundsicherungsstellen, die Aktivierung und Förderung besonders schwer in Erwerbsarbeit integrierbarer älterer Hilfebezieher zu unterlassen, so dass diese nach einem Jahr

\footnotetext{
${ }^{18}$ Vgl. zu dem Risiko, dass eine streng an den Maßstäben von Wirkung und Wirtschaftlichkeit orientierte Arbeitsmarktpolitik Personen mit schweren Vermittlungshemmnissen tendenziell ausschließt, die Studie von Hielscher und Ochs (2009). Empirische Belege für eine bevorzugte Förderung und Vermittlung von Hilfeempfängern mit geringeren Eingliederungshemmnissen durch die Mitarbeiter der SGB-II-Träger finden sich in den Fallstudien von A. Yollu-Tok (2010, S. 158, 179 f.).
}

ohne Stellenangebot nicht mehr in der Arbeitslosenstatistik erscheinen (Dietz et al. 2008, S. 73). Zum anderen sind beschäftigungslose Bezieher von Arbeitslosengeld II nur noch bis zum vollendeten 63 . Lebensjahr vor einem erzwungenen Übergang in Altersrente geschützt; danach müssen sie so bald wie möglich eine Altersrente auch mit Abschlägen in Anspruch nehmen, da diese gegenüber der steuerfinanzierten Fürsorgeleistung grundsätzlich vorrangig ist (Paragraph 12a Satz 2 Nr. 1 SGB II). Bis 2007 hingegen waren Hilfebezieher erst dann zur Beantragung ihrer Altersrente gezwungen, wenn sie die Altersgrenze für deren abschlagsfreien Bezug erreicht hatten, also in der Regel das vollendete 65. Lebensjahr.

Dass auch langzeitarbeitslosen Älteren Schritt für Schritt der Weg zurück in das Berufsleben gebahnt werden kann und entsprechende Investitionen in ihre Reintegration nicht von vornherein vergebens sind, zeigen die Erfahrungen aus dem 2005 gestarteten Bundesprogramm „Perspektive 50plus - Beschäftigungspakte für Ältere in den Regionen“, dessen Wirkungen auf Bundes- und regionaler Ebene evaluiert wurden (zu den Ergebnissen der bundesweiten Evaluation siehe Büttner et al. $2007^{19}$ und 2009). Das Programm fußt auf der Idee, einen engen und dauerhaften Verbund der Grundsicherungsstellen mit den wichtigsten arbeitsmarktrelevanten Akteuren in einer Region, wie den Kammern und Wirtschaftsverbänden, Gewerkschaften, Sozialverbänden, Kirchen und lokalen Politikern, zu schmieden. Die Partner dieses Beschäftigungspakts verpflichten sich dem gemeinsamen Ziel, die Arbeitsmarktchancen älterer Langzeitarbeitsloser in der Region zu verbessern, indem sie jeweils ihre spezifischen Einfluss- und Handlungsmöglichkeiten nutzen und koordiniert zusammenarbeiten. Das Programm ist richtungsweisend, weil es auf innovative, individuell passgenaue und die gesundheitlichen und psychosozialen Probleme der Betroffenen einbeziehende Integrationsstrategien setzt und zugleich ein Schwergewicht auf die Ansprache und enge Zusammenarbeit mit den (potentiell) einstellenden Betrieben legt. Um neuartige Wege der Eingliederung älterer Langzeitarbeitsloser zu ermöglichen, wurde die Ausgestaltung der Förderung und die Mittelverwendung den Verantwortlichen vor Ort überlassen. Die Ergebnisse der wissenschaftlichen Begleitung für die erste Programmphase (2005-2007) sind ermutigend. So wurde das Bundesprogramm von den befragten älteren Teilnehmern und den auskunftsbereiten Betrieben überwiegend sehr positiv beurteilt. Mehr als ein Drittel der Teilnehmer übten nach Beendigung des Programms eine Tätigkeit auf

\footnotetext{
${ }^{19}$ Büttner, R., Knuth, M., Neuffer, S., Neukirch, S., Schweer, O., Stegmann, T.: Evaluation der ersten Phase des Bundesprogramms „Perspektive 50plus - Beschäftigungspakte für Ältere in den Regionen“ (2005-2007). Endbericht, 10. Dezember 2007. Institut Arbeit und Qualifikation der Universität Duisburg-Essen, Gelsenkirchen, Duisburg, unveröffentlichte Arbeit (2007).
} 
dem ersten Arbeitsmarkt aus ${ }^{20}$ und gemessen an den durchschnittlichen teilnehmerbezogenen Kosten pro Integration in den ersten Arbeitsmarkt war es bislang auch ,kein übermäßig teures Programm“ (Büttner et al. 2007, S. 175, siehe Fußnote 19). Allerdings entfalteten die Öffentlichkeitsarbeit und Unternehmensansprache durch die Pakte in der ersten Programmphase noch keine Breitenwirkung: Das Beschäftigungsniveau Älterer in den betreffenden Regionen erhöhte sich durch das Bundesprogramm zunächst nicht.

Mit Blick auf die Erfahrungen anderer Länder belegt eine Auswertung von mehr als 250 Studien aus verschiedenen europäischen Staaten, dass Aktivierungsprogramme für Menschen mit multiplen Problemlagen in Hinblick auf die unmittelbare Integration in reguläre Beschäftigung zwar weniger wirksam sind als für Arbeitsuchende ohne besondere Vermittlungshemmnisse. Sie schaffen aber für die große Mehrheit der Teilnehmer erst die Bedingungen für eine spätere Erwerbsintegration, indem sie zur Bewältigung psychosozialer und gesundheitlicher Probleme beitragen, Selbstvertrauen stärken und Handlungsmöglichkeiten durch neu erlernte Fähigkeiten erweitern (Fromm und Sproß 2008, S. 103-105). Erfolgreich sind solche Maßnahmen allerdings nur dann, ,,wenn die Programme auch als sinnvoll und nicht als Instrument der Repression erlebt werden. (...) Eine wichtige Voraussetzung (...) ist demnach ein Fallmanger, der auf die Interessen und Bedürfnisse der Klienten eingeht und sie nicht zur Teilnahme an Maßnahmen zwingt, die nicht als sinnvoll erlebt werden" (Fromm und Sproß 2008, S. 105). Dieser Befund deckt sich mit den Ergebnissen der vorliegenden Studie, wonach sich ein Viertel der untersuchten Nutzer des ELB trotz grundsätzlicher Arbeitswilligkeit allein deshalb für den erleichterten Leistungsbezug entschieden haben, weil sie bestimmte Mindestanforderungen an einen Arbeitsplatz stellten oder weil sie nicht in Maßnahmen gedrängt werden wollten.

Hingegen erscheint eine Aktivierungspolitik, die in erster Linie auf Zwangselemente und finanzielle Kürzungen setzt, die den Arbeitslosen und der Öffentlichkeit signalisiert, dass die Ursachen der Arbeitslosigkeit primär in den Defiziten der Beschäftigungslosen selbst begründen liegen und die auf eine maßgeschneiderte Förderung und Mitbestimmung der Betroffenen verzichtet, als nicht geeignet, Personen mit besonderen Vermittlungshemmnissen nachhaltig in den Arbeitsmarkt zu integrieren. Stattdessen besteht die Gefahr, dass eine solche Ausgestaltung aktivierender Arbeitsmarktpolitik das Armutsrisiko und die soziale Exklusion der

\footnotetext{
${ }^{20}$ Eine Evaluation der Eingliederungseffekte des Bundesprogramms auf Basis von Teilnehmer- und Kontrollgruppen scheiterte an dem Fehlen eines Indikators für die Programmteilnahme in den Statistikdaten der Bundesagentur für Arbeit, so dass nicht abgeschätzt werden kann, wie viele der betreuten Älteren auch ohne die Unterstützung der Beschäftigungspakte eine Arbeit aufgenommen hätten (Büttner et al. 2007, S. 15, siehe vorherige Fußnote).
}

Betroffenen erhöht, ihre gesundheitlichen und psychischen Probleme verschärft und damit Beschäftigungsbarrieren vergrößert satt reduziert. ${ }^{21}$ Auch wenn die aktive Arbeitsförderung für Personengruppen mit geringen Arbeitsmarktchancen auf den ersten Blick als teuer erscheinen mag, können sich entsprechende Investitionen für eine sozial integrierende Gesellschaft durchaus lohnen.

\section{Kurzfassung}

Bis zum Jahr 2007 konnten sich ab 58-jährige Erwerbslose in Deutschland für den „Leistungsbezug unter erleichterten Voraussetzungen“ (ELB) nach Paragraph 428 Sozialgesetzbuch (SGB) III entscheiden: Sie konnten erklären, dass sie keine Arbeit mehr aufnehmen wollten, und waren dann von der Verpflichtung zur Arbeitssuche befreit. Arbeitslosengeld, Arbeitslosenhilfe oder Arbeitslosengeld II erhielten sie weiterhin in voller Höhe. Die Ausnahme der Älteren von einem Grundprinzip der aktivierenden Arbeitsmarktpolitik - der strikten Koppelung des Leistungsanspruchs an die Erwerbsbereitschaft - ist Ausdruck der Spannungen zwischen politischen Zielen und sozialen Realitäten: Einerseits soll der Vorruhestand zurückgedrängt werden, um das wachsende Arbeitskräftepotenzial der Älteren besser zu nutzen, die Sozialsysteme finanziell zu stabilisieren und Altersarmut sowie sozialer Exklusion entgegenzuwirken. Andererseits stößt die berufliche Reintegration von älteren Arbeitslosen auf beträchtliche Probleme, sodass ihre Aktivierung unter betriebswirtschaftlichen Effizienzgesichtspunkten häufig als nicht lohnend erscheint, während umgekehrt ihr vorzeitiger Ruhestand unmittelbar die Arbeitslosenstatistik und Arbeitsvermittlung entlastet. Vor dem Hintergrund der politischen Bemühungen um eine stärkere Teilhabe Älterer am

\footnotetext{
${ }^{21}$ Ein entsprechendes Fazit ziehen auch G. Mohr und P. Richter (2008, S. 31 f.) auf Basis des aktuellen Forschungsstandes zu den psychosozialen Auswirkungen von Erwerbslosigkeit: Sofern Aktivierungsmaßnahmen darauf ausgerichtet seien, die meist ohnehin ausgeprägte Arbeitsorientierung von Erwerbslosen noch weiter zu verstärken, die Suchanstrengungen der Betroffenen trotz geringer Erfolgsaussichten $\mathrm{zu}$ intensivieren und Arbeitslose zu einer fast unbeschränkten Konzessionsbereitschaft zu zwingen, seien diese Interventionen ,geradezu als Behandlungsfehler" anzusehen, da sie die psychischen Ressourcen der Erwerbslosen zusätzlich verminderten. Zu einer kritischen Bewertung dieser Form der Aktivierungspolitik gelangen ebenfalls P. Bescherer, S. Röbenack und K. Schierhorn (2008, S. 23 f.) auf Grundlage der Befunde einer qualitativen Studie zur Erwerbsorientierung von Beziehern des Arbeitslosengelds II. Demnach liefen die verschärften Zumutbarkeitsregelungen weitgehend ins Leere, weil sie bei den mehrheitlich ohnehin stark erwerbsorientierten Hilfebeziehern „offene Türen“ einrennen. Vgl. dazu auch Frerichs und Naegele 2008, S. 197-199 sowie Hielscher und Ochs 2009 für Deutschland; Dean et al. 2003, S. 24, Hirsch 2003, S. 27 f., Joyce et al. 2010 sowie McQuaid und Lindsay 2005 für Großbritannien; Rosdahl und Weise 2000, S. 178 f. für Dänemark; siehe dazu außerdem die Länder vergleichenden Untersuchungen von Gallie et al. 2003 sowie Konle-Seidl und Eichhorst 2008, S. 439-441.
} 
Arbeitsleben beschäftigt sich der Beitrag mit der Frage, aus welchen Gründen sich die überwiegende Mehrheit der älteren Arbeitslosen mit Hilfe des ELB vom Arbeitsmarkt zurückgezogen hat. Die empirische Untersuchung beschränkt sich dabei auf die meist langzeitarbeitslosen Bezieher von Arbeitslosengeld II.

Die Aussagekraft der hier präsentierten Analysen unterliegt allerdings einer Einschränkung: Da die Gründe für die Wahl des ELB retrospektiv erhoben worden sind, können nachträgliche Rationalisierungsversuche die tatsächlichen Handlungsmotive bei einem Teil der Befragten verdeckt haben. Dabei ist angesichts der sensitiven Fragen nach Erwerbsbereitschaft und Arbeitswilligkeit insbesondere mit Verzerrungen durch sozial erwünschte Antworten zu rechnen. Die Untersuchung des Arbeitsmarktverhaltens älterer Arbeitsloser anhand der Regelung des ELB scheint aber ein möglicher Weg zu sein, um solche Verzerrungen zumindest zu begrenzen, da die Bereitschaft, fehlende Arbeitswilligkeit im Interview offenzulegen, bei denjenigen älteren Leistungsempfängern höher sein dürfte, denen der Gesetzgeber explizit das Recht zugebilligt hat, nicht mehr arbeiten zu wollen. Ergänzende Auswertungen stützten diese Vermutung, so dass davon ausgegangen wird, dass die Angaben der Befragten wenigstens in der Tendenz ein zutreffendes Bild von den Gründen für ihre Abkehr vom Arbeitsmarkt zeichnen.

Hinsichtlich des Übergangs älterer Arbeitsloser in den Vorruhestand ergeben sich aus mikroökonomischen und soziologisch-psychologischen Erklärungsansätzen unterschiedliche Interpretationen: Aus mikroökonomischer Sicht erscheint die Wahl des ELB als das Resultat der nutzenmaximierenden Arbeitsangebotsentscheidung eines Individuums, das sich auch aufgrund der negativen Arbeitsanreize des sozialen Sicherungssystems für Freizeit und gegen Erwerbsarbeit entscheidet. Demgegenüber ist nach Ansätzen und Befunden aus der soziologisch-psychologischen Arbeitslosenforschung zu erwarten, dass die älteren Erwerbslosen unabhängig von materiellen Arbeitsanreizen auch nach längerer Arbeitslosigkeit mehrheitlich noch eine starke innere Bindung an Erwerbsarbeit aufweisen, sich aber wegen fehlender Beschäftigungsaussichten resigniert vom Arbeitsmarkt zurückziehen, um die motivationale Inkongruenz zwischen Erwerbswunsch und fehlender Erwerbsmöglichkeit zu beseitigen.

Die empirische Analyse zeigt auf Basis einer Befragung von Beziehern des Arbeitslosengelds II, dass der Rückzug älterer Langzeitarbeitsloser vom Arbeitsmarkt nicht in erster Linie in der mangelnden Erwerbsmotivation der Betroffenen gründet: Nur rund ein Achtel der befragten Nutzer wählte die Ausstiegsoption, weil sie tatsächlich nicht mehr arbeiten wollten, und für ein weiteres Viertel der Nutzer hatte die Erwerbs- und Mitwirkungsbereitschaft klare Grenzen: Sie optierten für den ELB hauptsächlich deshalb, weil sie nicht jede zumutbare Arbeit annehmen, kontinuierlich Bewerbungen nachweisen oder an angebotenen Fördermaßnahmen teilnehmen wollten. Die meisten der befragten Älteren hingegen wollten ihrem Selbstverständnis nach wieder in das Arbeitsleben zurückkehren, insbesondere dann, wenn sie noch keine ausreichende Absicherung für das Alter erreicht hatten, wie die Ergebnisse der multivariaten Analyse belegen. Der vielfache Wechsel älterer Erwerbsloser in den Vorruhestand ist daher nicht in erster Linie das Ergebnis nutzenmaximierender Entscheidungen zugunsten von Freizeit und gegen Erwerbsarbeit gewesen. Er kann deshalb auch nicht vorrangig als Problem falscher sozialrechtlicher Anreizwirkungen und insbesondere der negativen Arbeitsanreize des ELB selbst angesehen werden. Stattdessen scheint der wichtigste Grund für den Ausstieg der Älteren in den fehlenden Erwerbsperspektiven der Betroffenen zu liegen: Für mehr als $60 \%$ der Nutzer war die Einschätzung ausschlaggebend, den beruflichen Anforderungen aus gesundheitlichen oder anderen Gründen nicht mehr gewachsen zu sein, auf dem Arbeitsmarkt keine Chancen mehr zu haben und auch von der betreuenden Stelle keine ausreichende Hilfe und Förderung zu erhalten. Insbesondere für Männer aus Ostdeutschland, allen voran aber für ostdeutsche Frauen waren die Perspektivlosigkeit auf dem Arbeitsmarkt und ihre geringen Erwartungen an die Unterstützungsbereitschaft und -möglichkeiten der Arbeitsverwaltung der dominierende Rückzugsgrund. Für die wirksame Aktivierung und Integration älterer Langzeiterwerbsloser dürfte es daher weniger auf ein strikteres „Fordern“ als vielmehr auf ein besseres „Fördern“ und geeignete Beschäftigungsmöglichkeiten ankommen.

\section{Executive summary}

In Germany, until the end of 2007, unemployed persons aged 58 and over could opt for the Facilitated Receipt of Benefits (FRB), provided by Section 428 of the Social Code III. In order to benefit from this scheme, they simply had to declare that they did not want to take up work anymore and were thus exempt from the obligation to seek employment. Nevertheless, they were still entitled to receive full Unemployment Insurance Benefit, Unemployment Assistance or Unemployment Benefit II. The exemption of this group from a fundamental principle of labour market policy - the strict linkage between entitlement to benefits and willingness to work-evinces important tensions between political aims and social realities: on the one hand, early retirement should be restricted to realise the labour force potential of elderly workers, thereby stabilising the social security system and reducing dependencies and social exclusion in old-age. On the other hand, the reintegration of older unemployed individuals brings about considerable problems that all too often 
make their activation appear unprofitable from the efficiency perspective - by contrast, early retirement reduces visible unemployment as well as the amount of work imposed on employment agencies. In the light of the political goal of an increased labour market participation of older workers this paper deals with the reasons why the majority of older unemployed individuals left the labour force by making use of the FRB. The empirical analysis is restricted to the mostly long-term unemployed recipients of Unemployment Benefit II.

The validity of this study is affected by a methodical problem: The survey data about the motivation for choosing the FRB were collected retrospectively, which is why the respondents might have attempted to rationalise their decision ex post. For this reason the real motives might be disguised in many cases. Given that survey questions concerning the willingness to work and job search activities are considered sensitive, a social desirability bias must be taken into account. However, as FRB payment recipients are legally entitled to cease seeking employment, they can more likely be expected to reveal a lack of propensity to work. The findings of a supplementary study support this assumption: the self-reported motives can be supposed to draw a sufficiently realistic picture of why older long-term unemployed persons withdraw from the labour market.

The paper focuses on two possible interpretations coming from microeconomic and socio-psychological approaches. From a microeconomic perspective early retirement is regarded as the result of a utility-maximising labour supply decision: the older unemployed individual opts for leisure time and against gainful employment chiefly because of the disincentives of the social security system. By contrast, theories and empirical findings concerning the psycho-social effects of long-term unemployment suggest that-regardless of monetary incentives to work-the majority of older benefit recipients has a strong intrinsic commitment to gainful employment even after a long period of unemployment. They retire with feelings of resignation in order to remedy the motivational incongruence between their willingness to work and the lack of employment opportunities.

The empirical analysis based on a survey of recipients of Unemployment Benefit II shows that the main reason for retirement of the older long-term unemployed was not a lack of motivation to accept gainful employment. Only about one-eighth of the interviewees explicitly stated unwillingness to work as a reason for their choice to retire. A further quarter displayed only a very limited willingness to work: this group primarily opted for the FRB because they would accept only certain jobs, did not want to apply repeatedly for jobs or participate in employment and training programmes. In contrast, the majority of the interviewed recipients of the FRB wanted to re-enter gainful employment. Multivariate regression analysis shows that this is in particular true for those who expect insufficient funds in old-age. It is thus not primarily a result of individual utility maximisation (by implementing a preference for leisure time over gainful employment) when long-term unemployed opt for early retirement. Therefore, this behaviour cannot be seen primarily as a consequence of the disincentives of the social security system and of the FRB in particular. Instead, the most important reason for the exit of older unemployed persons from the labour force can be found in their lack of employment prospects: more than 60 per cent of those participating in the FRB declared their inability to cope with the challenges of a job due to poor health or other impediments, stated that they did not have any labour market opportunities or claimed that the respective agency could not provide sufficient help and support. Especially for East German men, but first and foremost for women in East Germany, the lack of job prospects as well as little trust in the willingness and ability of the public employment service to provide effective assistance played a dominating role in the decision to retire. Consequently, improved support and appropriate employment opportunities can be expected to foster more effectively activation and integration of the older long-term unemployed than pressure alone.

Danksagung Ich danke den zwei anonymen Gutachtern/-innen der Zeitschrift für Arbeitsmarktforschung für ihre wichtigen Anmerkungen und Hinweise. Mein besonderer Dank gilt Martin Brussig (Institut Arbeit und Qualifikation IAQ, Universität Duisburg-Essen) für den fruchtbaren inhaltlichen Austausch.

\section{Literatur}

Auspurg, K.: Die Analyse sozialer Ungleichheit. Konzeptuelle Überlegungen und empirische Erkenntnisse. Dissertation, Univ. Konstanz, Fachbereich Geschichte und Soziologie, veröffentlicht im Konstanzer Online-Publikations-System (KOPS). http://kops.ub. uni-konstanz.de/volltexte/2010/12471/ (2010)

Beatty, C., Fothergill, S.: Moving older people into jobs: Incapacity Benefit, Labour's reforms and the job shortfall in the UK regions. In: Loretto, W., Vickerstaff, S., White, P. (Hrsg.) The Future for Older Workers. New perspectives, S. 65-87. Policy Press, Bristol (2007)

Bender, S., Koch, S., Meßmann, S., Walwei, U.: Was muten sich Arbeitslose zu? Lohnkonzessionen von ALG II-Empfängern. Soz. Fortschr. 57, 75-85 (2008)

Bescherer, P., Röbenack, S., Schierhorn, K.: Nach Hartz IV: Erwerbsorientierung von Arbeitslosen. Polit. Zeitgesch. 58, 19-24 (2008)

Beste, J., Bethmann, A., Trappmann, M.: Arbeitsmotivation und Konzessionsbereitschaft: ALG-II-Bezug ist nur selten ein Ruhekissen. IAB-Kurzbericht 15/2010. Institut für Arbeitsmarkt- und Berufsforschung, Nürnberg (2010)

Betzelt, S.: Hartz VI aus Gender-Sicht: Einige Befunde und viele offene Fragen. WSI-Mitt. 60, 298-304 (2007)

Börner, M.: Erfolgreiche Arbeitssuche. Personale und situative Determinanten des Arbeitsplatzsuchverhaltens. Eine empirische Untersuchung an Erwerbslosen, Erwerbstätigen und Arbeitgebern. Hagener Arbeiten zur Organisationspsychologie. Bd. 2. LIT Verlag, Münster (2001) 
Börsch-Supan, A.: Incentive effects of social security on labor force participation: evidence in Germany and across Europe. J. Public Econ. 78, 25-49 (2000)

Boockmann, B., Thomsen, S., Walter, T.: Aktivierung der erwerbsfähigen Hilfebedürftigen mit arbeitsmarktpolitischen Maßnahmen - wer wird gefördert? Empirische Ergebnisse auf Grundlage eines neuen Surveydatensatzes. AStA Wirtsch. Sozialstat. Arch. 4, 269-292 (2010)

Brauer, K.: „Er ging nicht in eine Berufsunfähigkeitsrente, er konnte einfach nicht mehr“. Perspektiven von Älteren in deutschen Unternehmen. In: Brauer, K., Korge, G. (Hrsg.) Perspektive 50plus? Theorie und Evaluation der Arbeitsmarktintegration Älterer. Alter(n) und Gesellschaft, Bd. 18, S. 87-113. VS-Verlag, Wiesbaden (2009)

Brauer, K., Clemens, W.: Die Arbeitsmarktintegration Älterer aus der Perspektive der Alter(n)ssoziologie. In: Brauer, K., Korge, G. (Hrsg.) Perspektive 50plus? Theorie und Evaluation der Arbeitsmarktintegration Älterer. Alter(n) und Gesellschaft, Bd. 18, S. 2540. VS-Verlag, Wiesbaden (2009)

Brauer, K., Korge, G.: „Perspektive 50plus“: Arbeitsmarktintegration Älterer als sozialpolitische Gestaltungs- und wissenschaftliche Forschungsaufgabe. Eine Einleitung. In: Brauer, K., Korge, G. (Hrsg.) Perspektive 50plus? Theorie und Evaluation der Arbeitsmarktintegration Älterer. Alter(n) und Gesellschaft, Bd. 18, S. 9-21. VS-Verlag, Wiesbaden (2009)

Brussig, M.: Fast die Hälfte aller neuen Altersrenten mit Abschlägen Quote weiterhin steigend. Probleme mit dem Anstieg der Altersgrenzen vor allem bei Arbeitslosen, aber auch bei Erwerbstätigen. Altersübergangs-Report 1/2010. Institut Arbeit und Qualifikation, Hans-Böckler-Stiftung, Duisburg, Düsseldorf (2010a)

Brussig, M.: Erwerbstätigkeit im Alter hängt vom Beruf ab. Ausdifferenzierung der Erwerbschancen vor allem nach dem 60. Lebensjahr, in einigen Berufen aber schon früher. AltersübergangsReport 5/2010. Institut Arbeit und Qualifikation, Hans-BöcklerStiftung, Duisburg, Düsseldorf (2010b)

Brussig, M.: Neueinstellungen im Alter: Tragen sie zu verlängerten Erwerbsbiografien bei? Altersübergangs-Report 3/2011. Institut Arbeit und Qualifikation, Hans-Böckler-Stiftung, Duisburg, Düsseldorf (2011)

Brussig, M., Stegmann, T.: Wer geht vorzeitig in Rente? Eine Analyse mit den Individualdaten des Versichertenrentenzugangs 2004. In: Deutsche Rentenversicherung Bund (Hrsg.) Erfahrungen und Perspektiven. Bericht vom dritten Workshop des Forschungsdatenzentrums der Rentenversicherung (FDZ-RV) vom 26. bis 28 . Juni 2006 in Bensheim. DRV-Schriften. Bd. 55/2006, S. 135-157. WDV, Bad Homburg (2007)

Brussig, M., Wojtkowski, S.: Mehr Ältere auf dem Arbeitsmarkt: Erwerbstätigkeit und Arbeitslosigkeit nehmen zu. AltersübergangsReport 3/2007. Institut Arbeit und Qualifikation, Hans-BöcklerStiftung, Duisburg, Düsseldorf (2007)

Brussig, M., Wübbeke, C.: Policy-making in ageing labour markets. The case of hidden early retirement in Germany. In: Kuhn, M., Ochsen, C. (Hrsg.) Labour Markets and Demographic Change. Demografischer Wandel - Hintergründe und Herausforderungen, S. 252-280. VS-Verlag, Wiesbaden (2009)

Büttner, R., Knuth, M., Schweer, O., Stegmann, T.: Der Beitrag des Bundesprogramms Perspektive 50plus zur Verbesserung der Situation älterer Langzeitarbeitsloser. In: Behling, M., Huber, A., Staudinger, T. (Hrsg.) Perspektiven auf dem Arbeitsmarkt von morgen. Herausforderungen erkennen, Chancen nutzen, S. 32-46. Augsburg Integration Plus, Augsburg (2009)

Bundesagentur für Arbeit: Arbeitsmarkt 2005. Sondernummer der Amtlichen Nachrichten der Bundesagentur für Arbeit (ANBA). Bundesagentur für Arbeit, Nürnberg (2006)

Bundesagentur für Arbeit: Arbeitsmarkt 2006. Sondernummer der Amtlichen Nachrichten der Bundesagentur für Arbeit (ANBA). Bundesagentur für Arbeit, Nürnberg (2007)
Dean, H., MacNeill, V., Melrose, M.: Ready to work? Understanding the experiences of people with multiple problems and needs. Benefits 11, 19-25 (2003)

Deeke, A., Dietrich, H., Kruppe, T., Lott, M., Rauch, A., Stephan, G., Wolff, J.: Geförderte Qualifizierungsmaßnahmen in Deutschland. Aktuelle Evaluationsergebnisse im Überblick. Soz. Fortschr. 60, 196-203 (2011)

Dieck, M., Naegele, G.: „Neue Alte“ und alte soziale Ungleichheiten vernachlässigte Dimensionen in der Diskussion des Altersstrukturwandels. In: Naegele, G., Tews, H.P. (Hrsg.) Lebenslagen im Strukturwandel des Alters. Alternde Gesellschaft - Folgen für die Politik, S. 43-60. Westdeutscher Verlag, Opladen (1993)

Dietz, M., Klinger, S., Stephan, G., Walwei, U., Wolff, J., Wübbeke, C.: Jüngste Arbeitsmarktreformen: Schöne Aussichten für ältere Arbeitnehmer? IAB-Forum 4, 70-75 (2008)

Drobnič, S.: Retirement timing in Germany: the impact of household characteristics. Int. J. Sociol. 32, 75-102 (2002)

Ebbinghaus, B.: Reforming Early Retirement in Europe, Japan and the USA. Oxford University Press, Oxford (2006)

Eichhorst, W.: Beschäftigung Älterer in Deutschland: Der unvollständige Paradigmenwechsel. Z. Sozialreform 52, 101-123 (2006)

Franz, W.: Arbeitsmarktökonomik, 6 Aufl. Springer, Berlin (2006)

Frerichs, F., Naegele, G.: Active ageing in employment - prospects and policy approaches in Germany. In: Taylor, P. (Hrsg.) Ageing Labour Forces, S. 174. Edward Elgar, Cheltenham (2008)

Frerichs, F., Taylor, P.: The Greying of the Labour Market: What can Britain and Germany Learn from each Other? Anglo-German Foundation for the Study of Industrial Society, London (2005)

Fromm, S., Sproß, C.: Die Aktivierung erwerbsfähiger Hilfeempfänger. Programme, Teilnehmer, Effekte im internationalen Vergleich. IAB-Forschungsbericht, Bd. 1/2008. Institut für Arbeitsmarkt- und Berufsforschung, Nürnberg (2008)

Gallie, D., Pangam, S., Jacobs, S.: Unemployment, poverty and social isolation: is there a vicious circle of social exclusion? Eur. Soc. $\mathbf{5}$, $1-32(2003)$

Giddens, A.: Modernity and Self-Identity: Self and Society in the Late Modern Age. Polity, Cambridge (1991)

Goffman, E.: Stigma. Notes on the Management of Spoiled Identity. Prentice-Hall, Englewood Cliffs (1963)

Grawe, K.: Psychological Therapy. Hogrefe, Seattle (2004)

Gruber, J., Wise, D.A.: Social security programs and retirement around the World: micro estimation. NBER working paper 9407, National Bureau of Economic Research, Cambridge, Massachusetts (2002)

Gundert, S., Hohendanner, C.: Leiharbeit und befristete Beschäftigung: Soziale Teilhabe ist eine Frage von stabilen Jobs. IABKurzbericht, Bd. 4/2011. Institut für Arbeitsmarkt- und Berufsforschung, Nürnberg (2011)

Haupt, H., Liebscher, R., Winkler, G.: Ältere Arbeitnehmer/innen in Ostdeutschland - Lebenslagen und Lebensverhältnisse, Werte und Handlungsorientierungen. Umbruch - Beiträge zur Sozialen Transformation in den alten und neuen Bundesländern, Bd. 19. Trafo Verlag, Berlin (2005)

Heinemann, S., Gartner, H., Jozwiak, E.: Arbeitsförderung für Langzeitarbeitslose. Erste Befunde zu Eingliederungsleistungen des SGB III im Rechtskreis SGB II. IAB-Forschungsbericht Bd. 3/2006. Institut für Arbeitsmarkt- und Berufsforschung, Nürnberg (2006)

Henkens, K., Sprengers, M., Tazelaar, F.: Unemployment and the older worker in the Netherlands: re-entry into the labour force or resignation. Ageing Soc. 16, 561-578 (1996)

Hetschko, C., Knabe, A., Schöb, R.: Changing identity: retiring from Unemployment. SOEPpapers 399/2011. DIW, Berlin (2011)

Hielscher, V., Ochs, P.: Arbeitslose als Kunden? Beratungsgespräche der Arbeitsvermittlung zwischen Druck und Dialog. Sigma, Berlin (2009) 
Hirsch, D.: Crossroads After 50. Improving Choices in Work and Retirement. Joseph Rowntree Foundation, York (2003)

Humphrey, A., Costigan, P., Pickering, K., Stratford, N., Barnes, M.: Factors affecting the labour market participation of older workers. Research report 200, Department of work and pensions, Her Majesty's Stationery Office, Leeds, UK (2003)

Jabsen, A., Buchholz, S.: Increasing uncertainty in old age in Germany? The development of social inequality in later life since the mid-1980s. flexCAREER working paper. University of Bamberg, Bamberg (2009)

Jackson, P.R., Taylor, P.E.: Factors associated with employment status in later working life. Work Employ. Soc. 8, 553-567 (1994)

Jahoda, M.: Wieviel Arbeit braucht der Mensch? Arbeit und Arbeitslosigkeit im 20. Jahrhundert. Beltz, Weinheim (1983)

Joyce, K.E., Smith, K.E., Sullivan, C., Bambra, C.: 'Most of industry's shutting down up here': employability initiatives to tackle worklessness in areas of low labour market demand. Soc. Policy. Soc. 9, 337-353 (2010)

Kirchler, E.: Resigniert erstarren oder erfolgreich sein Schicksal schmieden? Veröffentlichung des Österreichischen Instituts für Arbeitsmarktpolitik, Bd. 36 (1991). Linz

Kistler, E., Hilpert, M.: Auswirkungen des demographischen Wandels auf Arbeit und Arbeitslosigkeit. Polit. Zeitgesch. 51, 5-13 (2001)

Knopf, D.: Alter zwischen Ent- und Verpflichtung. In: Institut für Soziale Infrastruktur; Bundesarbeitsgemeinschaft Seniorenbüros (Hrsg.) Grundsatzthemen der Freiwilligenarbeit. Theorie und Praxis des sozialen Engagements und seine Bedeutung für ältere Menschen, S. 69-84. Verlag Peter Wiehl, Stuttgart (2002)

Koch, S., Stephan, G., Walwei, U.: Workfare: Möglichkeiten und Grenzen. Z. Arb.markt Forsch. 38, 419-440 (2005)

Koller, B., Gruber, H.: Ältere Arbeitnehmer im Betrieb und als Stellenbewerber aus der Sicht der Personalverantwortlichen. Mitt. Arb.markt- Berufsforsch. 34, 479-505 (2001)

Konle-Seidl, R., Eichhorst, W.: Does activation work? In: Eichhorst, W., Kaufmann, O., Konle-Seidl, R. (Hrsg.) Bringing the Jobless into Work? Experiences with Activation Schemes in Europe and the US, S. 415-443. Springer, Berlin (2008)

Kraatz, S., Rhein, T., Sproß, C.: Internationaler Vergleich: Bei der Beschäftigung Älterer liegen andere Länder vorn. IAB-Kurzbericht 5/2006. Institut für Arbeitsmarkt- und Berufsforschung, Nürnberg (2006)

Krone, S.: Mit ,Best ager' auf Erfolgskurs - was sagen die Kund/innen dazu? In: Brauer, K., Korge, G. (Hrsg.) Perspektive 50plus? Theorie und Evaluation der Arbeitsmarktintegration Älterer. Alter(n) und Gesellschaft, Bd. 18, S. 263-278. VS-Verlag, Wiesbaden (2009)

Krumpal, I., Näher, A.: Entstehungsbedingungen sozial erwünschten Antwortverhaltens. Eine experimentelle Onlinestudie zum Einfluss des Wordings und des Kontexts bei unangenehmen Fragen. Soz. Welt 63, 65-89 (2012)

Lazarus, R.S., Launier, R.A.: Stressbezogene Transaktionen zwischen Person und Umwelt. In: Nitsch, J.R. (Hrsg.) Stress. Theorien, Untersuchungen, Maßnahmen, S. 213-259. Huber, Bern (1981)

Lehr, U.: Die Wiederentdeckung der Älteren in den Unternehmen: rechtliche und personalpolitische Überlegungen zum Thema „Alter" (Vortrag am 21.6.2007 in Passau). Neue Z. Arbeitsrecht 25 (Beilage 1/2008 zu Heft 7/2008), 3-8 (2008)

McQuaid, R., Lindsay, C.: The concept of employability. Urban Stud. 42, 197-219 (2005)

Mika, T., Baumann, J.: Soziale Konsequenzen der Abschaffung des Vorruhestands für Langzeitarbeitslose. WSI-Mitt. 61, 605-611 (2008)

Mohr, G., Richter, P.: Psychosoziale Folgen von Erwerbslosigkeit und Intervention. Polit. Zeitgesch. 58, 25-32 (2008)

Motel-Klingebiel, A., Engstler, H.: Einkommensdynamik beim Übergang in den Ruhestand. In: Künemund, H., Schroeter, K.R.
(Hrsg.) Soziale Ungleichheit und kulturelle Unterschiede in Lebenslauf und Alter, S. 141-159. VS Verlag, Wiesbaden (2008)

Mümken, S., Brussig, M., Knuth, M.: Vor der Anhebung des Renteneintrittsalters: Arbeitslosigkeit am Ende des Erwerbslebens. Wenig Förderung älterer Arbeitsloser. Soz. Sicherh. 59, 51-59 (2011)

Nordenmark, M., Strandh, M.: Towards a sociological understanding of mental well-being among the unemployed: the role of economic and psychosocial factors. Sociol. 33, 577-597 (1999)

Paul, K.I., Geithner, E., Moser, K.: Latent deprivation among people who are employed, unemployed, or out of the labour force. J. Psychol.: Interdiscip. Appl. 143, 477-491 (2009)

Paul, K.I., Moser, K.: Incongruence as an explanation for the negative mental health effects of unemployment: Meta-analytic evidence. J. Occup. Organ. Psychol. 79, 595-621 (2006)

Porcellato, L., Carmichael, F., Hulme, C., Ingham, B., Prashar, A.: Giving older workers a voice: constraints on the employment of older people in the North West of England. Work Employ. Soc. 24, 85-103 (2010)

Promberger, M.: Arbeit, Arbeitslosigkeit und soziale Integration. Polit. Zeitgesch. 58, 7-15 (2008)

Promberger, M., Wenzel, U., Pfeiffer, S., Hacket, A., Hirseland, A.: Beschäftigungsfähigkeit, Arbeitsvermögen und Arbeitslosigkeit. WSI-Mitt. 61, 70-76 (2008)

Radl, J.: Individuelle Determinanten des Renteneintrittsalters. Eine empirische Analyse von Übergängen in den Ruhestand. Z. Soziol. 36, 43-64 (2007)

Riach, K., Loretto, W.: Identity work and the 'unemployed' worker: age, disability and the lived experience of the older unemployed. Work Employ. Soc. 23, 102-119 (2009)

Roberts, I.: Taking age out of the workplace: putting older workers back in? Work Employ. Soc. 20, 67-86 (2006)

Rodriguez, Y.G.: Learned helplessness or expectancy-value? A psychological model for describing the experiences of different categories of unemployed people. J. Adolesc. 20, 321-332 (1997)

Rosdahl, A., Weise, H.: When all must be active: workfare in Denmark. In: Loedemel, I., Trickey, H. (Hrsg.) An Offer You Can't Refuse: Welfare to Work in Seven Countries, S. 159-180. Policy Press, Bristol (2000)

Seligman, M.E.P.: Helplessness. WH Freeman, San Francisco (1975)

Sessions, J.G.: Unemployment stigma and multiple labour market equilibria: a social-psychological interpretation of hysteresis. Labour 8, 355-375 (1994)

Statistik der Bundesagentur für Arbeit: Grundsicherung für Arbeitsuchende: Verweildauern von Hilfebedürftigen, Februar 2010. http://statistik.arbeitsagentur.de/Statischer-Content/StatistischeAnalysen/Statistische-Sonderberichte/Generische-Publikationen/ SGBII/Sonderbericht-Verweildauer.pdf. (2010). Zugegriffen am 2. August 2011

Statistik der Bundesagentur für Arbeit: Analytikreport der Statistik: Analyse des Arbeitsmarktes für Ältere ab 50 Jahren, Juni 2011. http://statistik.arbeitsagentur.de/Statischer-Content/StatistischeAnalysen/Analytikreports/Zentrale-Analytikreports/MonatlicheAnalytikreports/Generische-Publikationen/Analyse-Arbeitsmarkt -Aeltere/Analyse-Arbeitsmarkt-Aeltere-201106.pdf (2011). Zugegriffen am 2. August 2011

Tisch, A.: Kundenzufriedenheit im SGB II: Arbeitsvermittler im Urteil der ALG-II-Empfänger. IAB-Kurzbericht, Bd. 7/2010. Institut für Arbeitsmarkt- und Berufsforschung, Nürnberg (2010)

Trischler, F., Kistler, E.: Gute Erwerbsbiographien. Arbeitspapier 1: Erwerbsverläufe im Wandel. Hans-Böckler-Stiftung, Internationales Institut für Empirische Sozialökonomie, Stadtbergen (2010a)

Trischler, F., Kistler, E.: Gute Erwerbsbiographien. Arbeitspapier 2: Arbeitsbedingungen und Erwerbsverlauf. Hans-Böckler-Stiftung, Internationales Institut für Empirische Sozialökonomie, Stadtbergen $(2010 b)$ 
van Dyk, S., Lessenich, S.: „Junge Alte“: Vom Aufstieg und Wandel einer Sozialfigur. In: van Dyk, S., Lessenich, S. (Hrsg.) Die jungen Alten. Analysen einer neuen Sozialfigur, S. 11-48. Campus, Frankfurt am Main (2009)

Walwei, U.: Arbeitsmarktchancen Älterer. Empirische Befunde und Perspektiven. Dtsch. Rentenversicher. 65, 421-433 (2010)

Warr, P.B.: Work, Unemployment, and Mental Health. Clarendon, Oxford (1987)

Wübbeke, C.: Der Übergang in den Rentenbezug im Spannungsfeld betrieblicher Personal- und staatlicher Sozialpolitik. Beiträge zur Arbeitsmarkt- und Berufsforschung 290.1. Institut für Arbeitsmarkt- und Berufsforschung, Nürnberg (2005)
Yollu-Tok, A.: Die fehlende Akzeptanz von Hartz IV. Eine Realanalyse individuellen Verhaltens jenseits des Homo oeconomicus Modells. Nomos, Baden-Baden (2010)

Christina Wübbeke ist wissenschaftliche Mitarbeiterin im Forschungsbereich E2 „Erwerbslosigkeit und Teilhabe“ am Institut für Arbeitsmarkt- und Berufsforschung (IAB). Ihr aktueller Forschungsschwerpunkt liegt auf der beruflichen Eingliederung und sozialen Sicherung älterer Arbeitslosengeld-II-Empfänger. 